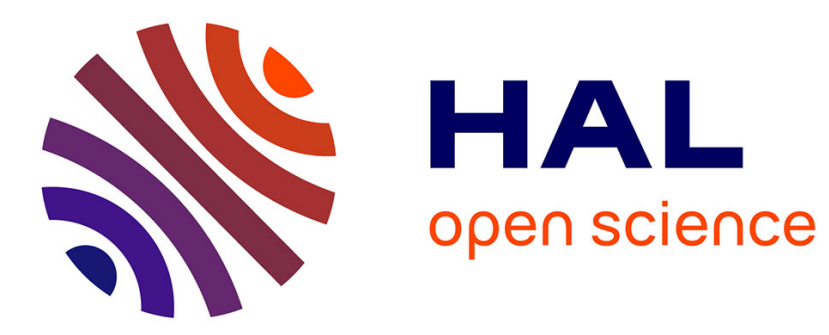

\title{
Blind hyperspectral unmixing using an Extended Linear Mixing Model to address spectral variability
}

\author{
Lucas Drumetz, Miguel Angel Veganzones, Simon Henrot, Ronald Phlypo,
} Jocelyn Chanussot, Christian Jutten

\section{- To cite this version:}

Lucas Drumetz, Miguel Angel Veganzones, Simon Henrot, Ronald Phlypo, Jocelyn Chanussot, et al.. Blind hyperspectral unmixing using an Extended Linear Mixing Model to address spectral variability. IEEE Transactions on Image Processing, 2016, IEEE Transactions on Image Processing, 25 (8), pp.3890-3905. 10.1109/TIP.2016.2579259 . hal-01336279

\section{HAL Id: hal-01336279 \\ https://hal.science/hal-01336279}

Submitted on 22 Jun 2016

HAL is a multi-disciplinary open access archive for the deposit and dissemination of scientific research documents, whether they are published or not. The documents may come from teaching and research institutions in France or abroad, or from public or private research centers.
L'archive ouverte pluridisciplinaire HAL, est destinée au dépôt et à la diffusion de documents scientifiques de niveau recherche, publiés ou non, émanant des établissements d'enseignement et de recherche français ou étrangers, des laboratoires publics ou privés. 


\title{
Blind hyperspectral unmixing using an Extended Linear Mixing Model to address spectral variability
}

\author{
Lucas Drumetz, Miguel-Angel Veganzones, Member, IEEE, Simon Henrot, Ronald Phlypo, Member, IEEE, \\ Jocelyn Chanussot, Fellow, IEEE, and Christian Jutten, Fellow, IEEE
}

\begin{abstract}
Spectral Unmixing is one of the main research topics in hyperspectral imaging. It can be formulated as a source separation problem whose goal is to recover the spectral signatures of the materials present in the observed scene (called endmembers) as well as their relative proportions (called fractional abundances), and this for every pixel in the image. A Linear Mixture Model is often used for its simplicity and ease of use but it implicitly assumes that a single spectrum can be completely representative of a material. However, in many scenarios, this assumption does not hold since many factors, such as illumination conditions and intrinsic variability of the endmembers, induce modifications on the spectral signatures of the materials. In this paper, we propose an algorithm to unmix hyperspectral data using a recently proposed Extended Linear Mixing Model. The proposed approach allows a pixelwise spatially coherent local variation of the endmembers, leading to scaled versions of reference endmembers. We also show that the classic nonnegative least squares, as well as other approaches to tackle spectral variability can be interpreted in the framework of this model. The results of the proposed algorithm on two different synthetic datasets, including one simulating the effect of topography on the measured reflectance through physical modelling, and on two real datasets, show that the proposed technique outperforms other methods aimed at addressing spectral variability, and can provide an accurate estimation of endmember variability along the scene thanks to the scaling factors estimation.
\end{abstract}

Index Terms-Hyperspectral imaging, remote sensing, blind source separation, spectral unmixing, spectral variability, spatial regularization, Alternating Direction Method of Multipliers.

\section{INTRODUCTION}

$\mathbf{H}$ YPERSPECTRAL imaging allows the acquisition of multivariate images containing information in hundreds of narrow and contiguous spectral bands, whose corresponding wavelengths typically range from the visible domain to the near infrared. In this type of data, each pixel of the image is a complete radiance or reflectance spectrum, augmenting

L. Drumetz, S. Henrot, and C. Jutten are with the Department Image and Signal (DIS), GIPSA-lab, Université Joseph Fourier, F-38402 Saint Martin d'Hères Cedex, France. Phone (e-mail: lucas.drumetz@gipsa-lab.fr; simon.henrot@gipsa-lab.fr; christian.jutten@gipsa-lab.fr)

M.A. Veganzones is with the Department Image and Signal (DIS), GIPSAlab, CNRS, F-38402 Saint Martin d'Hères Cedex, France. Phone (e-mail: miguel-angel.veganzones@gipsa-lab.fr

R. Phlypo and J. Chanussot are with the Department Image and Signal (DIS), GIPSA-lab, Grenoble-INP, F-38402 Saint Martin d'Hères Cedex, France(e-mail: ronald.phlypo@gipsa-lab.fr; jocelyn.chanussot@gipsa-lab.fr)

J. Chanussot is also with the Faculty of Electrical and Computer Engineering, University of Iceland, Reykjavik, Iceland.

J. Chanussot and C. Jutten are members of Institut Universitaire de France.

This work was partially supported by the European Research Council under the European Community's Seventh Framework Programme FP7/2007-2013, under Grant Agreements no.320684 (CHESS project) and no.320594 (DECODA project). considerably the spectral information contained in a pixel, with respect to RGB or multispectral images, for instance. This spectral information enables different types of applications [1], such as segmentation [2], classification [3], target detection [4]... However, the acquisition of data in many wavelengths comes with a lower spatial resolution than color or even multispectral images. Super-resolution algorithms (e.g. [5]) may improve the spatial resolution to a certain extent, but in any case, a single pixel cannot in general account for the interaction of light with only one material, present exclusively in the field of view of the sensor during the acquisition. These so-called mixed pixels make hyperspectral data interpretation much harder.

In this context, the analysis of such data can be seen as a blind (or semiblind) source separation problem (BSS), termed Spectral Unmixing (SU) [6]. Indeed, the amount of light arriving at the sensor can be seen as a mixture of the contributions coming from the interaction of light with all the materials in the sensor's field of view. Spectral Unmixing then aims at recovering, for each pixel, the materials composing it, as well as the proportions of each of them in the pixel. The former quantities are called the endmembers, while the latter are called fractional abundances. Because of its importance for data interpretability and analysis, SU has become one of the prime topics of hyperspectral data analysis. In most applications, a Linear Mixing Model (LMM) is assumed, considering that the contributions of each endmember sum up in a linear way [7]. Though the LMM has been used in the majority of works for a long time, in real situations several factors reveal some of its shortcomings. The main two factors hampering its efficiency have been identified as nonlinearities and spectral variability. In many real scenarios, the LMM is not a sufficiently good approximation of the real mixing process since many physical processes such as multiple scattering and intimate mixing contribute to the measured radiance or reflectance in a nonlinear fashion. Spectral variability is also an important fact that the LMM does not take into account: each endmember is implicitly assumed to be perfectly represented by a single spectral signature. This strong assumption often does not hold for real datasets since the measured radiance or reflectance of a material can significantly change depending for example on the geometry and topography of the scene, or because of atmospheric effects or even because of the intrinsic variability of the material, due to the variation of a hidden parameter (e.g. concentration of chlorophyll in vegetation).

Although nonlinear unmixing has recently received much attention in the community -providing new nonlinear mixing 
models and the corresponding SU algorithms [8], [9]-, spectral variability has been given less attention in comparison. Still, recent overviews of the spectral variability issue exist and summarize some of the methods taking it into consideration during the unmixing process [10], [11]. In each of these works a classification of the techniques used is proposed, the most general being the separation into two classes of methods, depending on whether variable endmembers are considered as sets or as probability distributions. The latter category models variability as a realization of some multivariate random variable, following a given mixture model, e.g. the Normal Compositional Model [12] or the Beta Compositional Model [13]. The unmixing process is then performed in a Bayesian estimation framework. This paper will focus on approaches in which each endmember variant is a part of a set comprising several instances of the same endmember. These endmember sets can be either known or unknown in advance. In the latter case, they have to be extracted from the data, resorting for instance to one of the many existing Endmember Extraction Algorithms (EEA) [7], [14], e.g. the Vertex Component Analysis (VCA) [15], the N-FINDR [16], or the Minimum Volume Simplex Analysis (MVSA) [17]. Whether the endmembers are assumed known or not, SU remains a (semi-)blind [18] or (un/semi-)supervised [19] source separation problem.

In the following, we introduce a new algorithm to perform SU taking into account the spatial information as well as spectral variability. We recently proposed the Extended Linear Mixing Model (ELMM) [20], which aims at addressing endmember variability while preserving the LMM framework. This model allows a pixelwise variation of the endmembers according to scaling factors. Another model addressing spectral variability was more recently proposed in [21], in which the variability is explained by an additive perturbation of the endmembers.

The remainder of this paper is organized as follows: Section II presents some of the mixing models and techniques related to the proposed approach, which is presented in Section III. Results on two synthetic datasets are presented in Section IV, results on two real datasets with different spatial and spectral resolutions and corresponding to different contexts are shown in Section $\mathrm{V}$ and some concluding remarks are gathered in Section VI.

\section{Spectral VARiability}

Let us denote a hyperspectral image as $\mathbf{X} \in \mathbb{R}^{L \times N}$ and $\mathbf{A} \in \mathbb{R}^{P \times N}$ the abundance matrix containing the abundance vectors for each pixel $\mathbf{a}_{k}(k=1, \cdots, N)$ in its columns. $L$ is the number of spectral bands, $N$ the number of pixels in the image, and $P$ is the number of endmembers considered. The different endmember matrices we will use will be denoted as $\mathbf{S} \in \mathbb{R}^{L \times P}$, possibly indexed by $k$ if allowed to vary spatially in the image. $\mathbf{E} \in \mathbb{R}^{L \times N}$ is an additive noise.

With these notations, the LMM writes, for the pixel $\mathbf{x}_{k} \in$ $\mathbb{R}^{L}$ :

$$
\mathbf{x}_{k}=\sum_{p=1}^{P} a_{p k} \mathbf{s}_{p}+\mathbf{e}_{k}=\mathbf{S} \mathbf{a}_{k}+\mathbf{e}_{k},
$$

with the abundance non-negativity constraint (ANC) $a_{p k} \geq 0$ since none of the physical quantities involved are supposed to be negative, and possibly the abundance sum-to-one constraint (ASC) $\sum_{p=1}^{P} a_{p k}=1, \forall k$, which means that each pixel has to be completely explained by the contributions of the endmembers. With the LMM and the two constraints, the data are assumed to lie inside a $(P-1)$-simplex whose vertices correspond to the endmembers. If the ASC is relaxed, the underlying model assumes the data lie inside a convex cone spanned by the endmembers.

The LMM can be written for all pixels in a matrix compact form as:

$$
\mathbf{X}=\mathbf{S A}+\mathbf{E} \text {. }
$$

In this section, some existing approaches to perform SU by taking into account spectral variability are presented. In particular, we introduce the Extended Linear Mixing Model.

\section{A. Spectral Bundles}

Spectral bundles [22] are a simple way to address endmember variability by building a candidate endmember dictionary. One possibility to do so is to run several times any EEA on randomly chosen subsets of the image. Each time the EEA is run, new instances of each endmember should be extracted. If the EEA uses the so-called pure pixel assumption, there has to be at least one pure pixel in each of these reduced datasets. If this assumption is too strong, it may be preferable to resort to a non pure pixel-based EEA, such as those reviewed in [14]. As the extracted sources suffer from the so-called permutation problem, i.e. the endmembers are not aligned from one subset to the other, a clustering step is required to group the candidate endmembers into classes. Then the abundances can be extracted in several ways, two of which are detailed in the following.

The sparse unmixing approach was first designed to see SU as a semi-supervised source separation problem in which a large dictionary of spectral signatures is known beforehand [23], but it can also be used if the dictionary is learnt from the data, as in the spectral bundles approach. By performing a sparse regression of the data on the dictionary, for instance using the SUnSAL algorithm [23], only a few signatures will be active in each pixel. To get global abundance maps, one only has to sum the contributions of every candidate in each endmember class. Another option to recover abundances once the spectral bundles have been extracted is to use the Fisher Discriminant Nullspace (FDN) approach [24]. This technique searches for a projection of the dataset onto a low-dimensional subspace such that intraclass variability of the endmember bundles is minimized and their interclass variability is maximized. In such a subspace, the data clouds formed by the candidate endmembers in each class are reduced to a mimimum volume. Finally, one only has to apply the traditional (Fully) Constrained Least Squares Unmixing ((F)CLSU) [25] to enforce the ANC (and possibly the ASC). In that case, a simple choice of the endmembers is to pick the centroids of each endmember bundle.

\section{B. Extended Linear Mixing Model}

Ideally, the endmembers should be allowed to vary in every pixel of the image, while the mixing process would remain linear. In [20], we proposed an Extended Linear Mixing Model (ELMM) to allow a pixelwise variation of each endmember: 


$$
\mathbf{x}_{k}=\sum_{p=1}^{P} a_{p k} f_{p k}\left(\mathbf{s}_{p}\right)+\mathbf{e}_{k},
$$

with some mappings $f_{p k}: \mathbb{R}^{L} \rightarrow \mathbb{R}^{L}$. As shown in [20], the spectral bundles approach can also be seen as a special case of (3), where the function $f_{p k}$ is implicitly modeled by some outcomes of this spectral variability function forming the endmember dictionary. Following our seminal idea and paper [20], authors in [21] proposed to model spectral variability with an additive perturbation of some reference endmembers. This clearly fits in the general framework we describe here.

If we assume $f_{p k}\left(\mathbf{s}_{0 p}\right)=\psi_{p k} \mathbf{s}_{0 p}$, i.e. that spectral variability consists in scalings of some reference endmembers $\mathbf{s}_{0 p}$, then Eq. (3) rewrites:

$\mathbf{x}_{k}=\sum_{p=1}^{P} a_{p k} \psi_{p k} \mathbf{s}_{0 p}+\mathbf{e}_{k}=\mathbf{S}_{0} \boldsymbol{\psi}_{k} \mathbf{a}_{k}+\mathbf{e}_{k}=\mathbf{S}_{0}\left(\boldsymbol{\psi}^{k} \circ \mathbf{a}_{k}\right)+\mathbf{e}_{k}$,

where $\psi_{k} \in \mathbb{R}^{P \times P}$ is a diagonal matrix with values $\psi_{p k} \geq 0$ on the diagonal, $\boldsymbol{\psi}^{k}=\operatorname{diag}\left(\boldsymbol{\psi}_{k}\right)$, and $\mathbf{S}_{0}$ is the reference endmember matrix, whose columns are the $\mathbf{s}_{0 p}$. The symbol - denotes the Schur-Hadamard (termwise) product. This can be written more compactly for all pixels in a matrix form:

$$
\mathbf{X}=\mathbf{S}_{0}(\mathbf{\Psi} \circ \mathbf{A})+\mathbf{E},
$$

where $\boldsymbol{\Psi}$ is a $\mathbb{R}^{P \times N}$ matrix gathering all the scaling factors for all pixels and all materials (its $k^{\text {th }}$ column is $\psi^{k}$ ).

The model of Eqs. (4) and (5) will be simply denoted hereafter as ELMM. With these definitions, an observed pixel is a linear combination of scaled versions of each endmember depending on the spatial dimension in the image. This special case of the model has appeared in the community in [15], [26] to explain variability due to the geometry of the scene (the spatial variation of the incidence, emergence and azimuth angles with the topography of the scene, see [27]) and can be considered as a good first approximation to reality. The use of the spectral angle as a metric to compare spectra also has the same rationale: it is insensitive to scalings and hence measures the dissimilarity in the shapes of the spectra, not their magnitude [28]. In the literature, to model shadow and brightness effects, a constant "shade" endmember is often considered, and its abundances are considered in the same way as for other materials [28]. However, using this trick boils down to scaling each pixel with the abundance of this shadow endmember, and then interpreting the remaining abundances for the other materials. In such a case, the ASC is not physically meaningful anymore since this endmember is not an actual material.

In order to further motivate the model advocated by Eq. (5), we manually selected two pure pixels of the same material in the image of the first real dataset used in the experiments of section V, and shown in Fig. 12. These pixels are part of the red rooftop on the northwestern part of the football field. The two pixels are part of two distinct facets of this pyramidal roof, which are very differently lit, since their orientation w.r.t. the sun is different. The spectral signatures are shown in Fig. 1. We performed a least squares regression in order to approximate the blue spectrum $\mathbf{x}_{1}$ by a scaled version of the red spectrum $\mathrm{x}_{2}$ :

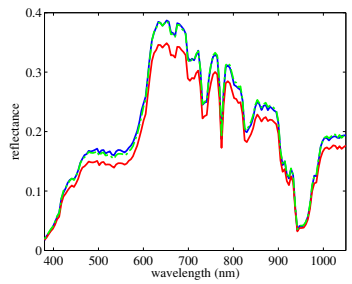

Fig. 1. Two pixels on different facets of the same red roof in the image of Fig 12 (red and blue). The result of the linear regression of the red spectrum on the blue one, using only a scaling factor (dashed green).

$$
\hat{\psi}=\underset{\psi}{\arg \min }\left\|\mathbf{x}_{1}-\psi \mathbf{x}_{2}\right\|_{2}^{2}
$$

The result of this regression (i.e. $\hat{\psi} \mathbf{x}_{2}$ ) is shown in green in Fig. 1. We can see that the fit is almost perfect, meaning that the variability between these two pure pixels can be approximated very well by a scaling factor, which is confirmed by the very high Pearson correlation coefficient between the original blue spectrum and its green regression $(r=0.9994)$.

The model of Eq. (5) has a simple geometric interpretation, as can be seen in Fig.2 (in a case where there are three endmembers). The data points are assumed to lie in a cone spanned by the reference endmembers. The scaling factors, combined with the ASC and ANC, constrain each pixel to lie in a simplex whose vertices are variants of the reference endmembers, situated on straight lines joining the origin and each of the reference endmembers, thus defining the simplex orientation in the cone.

More accurate radiative transfer-based models such as the Hapke model [29] are also used to account specifically for the variability due to topography and to the photometry of the materials, but due to their complexity, they are cumbersome to use in a SU context. In addition, [20] shows that the nonnegative or partially Constrained Least Squares Unmixing (CLSU) algorithm can be seen as addressing a special case of Eqs. (4) and (5) when the scaling factor is identical for each endmember and the ASC is assumed. Thus, one can recover the scaling factor (and hence the abundances) in a pixel by

$$
\sum_{p=1}^{P} \hat{\phi}_{k}^{p}=\sum_{p=1}^{P} a_{p k} \psi_{k}=\psi_{k} \sum_{p=1}^{P} a_{p k}=\psi_{k},
$$

where $\hat{\phi}_{k}^{p}$ is the quantity estimated by CLSU in pixel $k$ for endmember $p$. This scaled version of CLSU will be denoted by S-CLSU hereafter. To our knowledge, there exists no algorithm in the literature specifically designed to unmix hyperspectral data according to model of Eqs. (4) and (5).

\section{Proposed ApproACH}

We define a criterion (energy to minimize) to perform spectral unmixing using the ELMM (we denote by $\boldsymbol{\Psi}$ the scaling factors rearranged in a $\mathbb{R}^{P \times N}$ matrix and by $\underline{\mathbf{S}}=\left\{\mathbf{S}_{k}\right\}$ the collection of pixel-dependent endmember matrices):

$$
\begin{aligned}
\mathcal{J}(\mathbf{A}, \underline{\mathbf{S}}, \boldsymbol{\Psi}) & =\frac{1}{2} \sum_{k=1}^{N}\left(\left\|\mathbf{x}_{k}-\mathbf{S}_{k} \mathbf{a}_{k}\right\|_{2}^{2}+\lambda_{S}\left\|\mathbf{S}_{k}-\mathbf{S}_{0} \boldsymbol{\psi}_{k}\right\|_{F}^{2}\right) \\
& +\mathcal{R}(\mathbf{A})+\mathcal{R}(\mathbf{\Psi}) .
\end{aligned}
$$




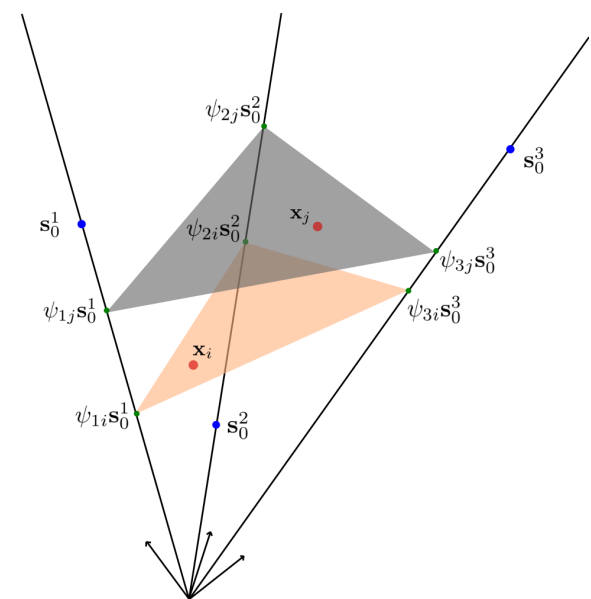

Fig. 2. Geometric interpretation of the ELMM in the case of three endmembers. In red are two data points, in blue are the reference endmembers and in green are the scaled versions for the two considered pixels.

Note that all quantities have to be positive and the abundances are subject to the ASC. The first two terms are the modelling terms, the ones which enforce the ELMM, more or less strictly depending on the value of $\lambda_{S} \cdot \mathcal{R}(\mathbf{A})$ and $\mathcal{R}(\boldsymbol{\Psi})$ are regularization terms applied to the abundances and the scaling factors, respectively. Their role is to incorporate the constraints on the variables and to enforce spatial smoothness. We chose the following regularization terms:

$$
\begin{aligned}
\mathcal{R}(\mathbf{A}) & =\lambda_{A} S R(\mathbf{A})+\mathcal{I}_{\mathbb{R}_{+}^{P \times N}}(\mathbf{A})+\boldsymbol{\mu}^{\top}\left(\mathbf{A}^{\top} \mathbb{1}_{P}-\mathbb{1}_{N}\right) \\
& =\lambda_{A}\left(\left\|\mathcal{H}_{h}(\mathbf{A})\right\|_{2,1}+\left\|\mathcal{H}_{v}(\mathbf{A})\right\|_{2,1}\right)+\mathcal{I}_{\mathbb{R}_{+}^{P \times N}}(\mathbf{A}) \\
& +\boldsymbol{\mu}^{\top}\left(\mathbf{A}^{\top} \mathbb{1}_{P}-\mathbb{1}_{N}\right) \\
& \mathcal{R}(\boldsymbol{\Psi})=\frac{\lambda_{\Psi}}{2}\left(\left\|\mathcal{H}_{h}(\boldsymbol{\Psi})\right\|_{F}^{2}+\left\|\mathcal{H}_{v}(\mathbf{\Psi})\right\|_{F}^{2}\right.
\end{aligned}
$$

where $\lambda_{A}, \lambda_{\Psi}$ are regularization parameters used to weigh the different terms in the optimization problem. $\boldsymbol{\mu} \in \mathbb{R}^{N}$ is a column vector of Lagrange mutlipliers for the ASC. $\mathcal{H}_{h}, \mathcal{H}_{v}$ : $\mathbb{R}^{P \times N} \rightarrow \mathbb{R}^{P \times N}$ are linear operators of horizontal and vertical gradients between adjacent pixels (acting separately on each material). $\mathcal{I}_{\mathbb{R}^{P \times N}}$ is the indicator function of the nonnegative orthant, and $\mathbb{1}_{(\cdot)}$ is a column vector of ones whose length is given in its index. SR stands for spatial regularization. We are simply using first order neighborhoods for the gradient computations. The spatial regularization on the abundances is the $\mathcal{L}_{1}$ norm of the channel-by-channel spatial regularizations, meaning each abundance map is smoothed independently. Using the definition of [30], it is a $\mathcal{L}_{2,1}$ mixed norm. Note that replacing this $\mathcal{L}_{2,1}$ norm by a $\mathcal{L}_{1,1}$ norm would result in a Total Variation (TV) penalization, similar to the one in [31]. In addition, only one line of code has to be changed to switch from the chosen regularization to a TV. This constraint on the abundances can be justified by the fact that the fractional abundances often exhibit spatially correlated patterns.

The ASC and ANC are also enforced by the last two terms. The Lagrange multipliers $\boldsymbol{\mu}$ also have to be optimized so that the ASC is enforced. Note that if we choose not to include the regularization on the abundances, they should still be subject to the ASC and ANC. For the scaling factors, we chose to simply use a spatial smoothness term, because the effects of certain causes of variability, such as the one due to topography and atmospheric effects, are likely to be spatially coherent. In Eq. (8), a reference endmember matrix $\mathbf{S}_{0}$ is required, and can be extracted by any EEA. Since we are estimating the actual abundances and not a product of the abundances and the scaling factors, the ASC is physically meaningful (except if nonlinearities are not negligible, but this is out of the scope of the present paper), and also acts as a calibration of the scaling factors $[32]^{1}$. Note that compared to Eqs. (4) and (5), a small perturbation on the model is allowed, depending on the value of $\lambda_{S}$. This regularization is also very useful to solve the ambiguity between the scaling factors and the abundances. As a matter of fact the introduction of the variables $\mathbf{S}_{k}$ allows to decouple the abundances and scaling factors. The update of either of these variables is no longer inversely proportional to the other (as it would be the case if we wanted so estimate the parameters by a least squares fit on Eq. (5)).

Since the problem we wish to solve is not convex w.r.t. all variables simultaneously, but convex w.r.t. each of them, we propose to find a stationary point by iteratively optimizing the criterion in an Alternating Nonnegative Least Squares (ANLS) way, following the outline given in Algorithm 1. The iterations terminate when the relative variations (measured using Frobenius norms) between consecutive iterates of $\mathbf{A}$, $\underline{\mathbf{S}}=\left\{\mathbf{S}_{k}\right\}$ and $\boldsymbol{\Psi}$ are below three tolerances $\epsilon_{A}, \epsilon_{S}$ and $\epsilon_{\Psi}$, respectively.

\section{Data: $\mathbf{X}, \mathbf{S}_{0}$}

Result: $\underline{\hat{\mathbf{S}}}, \hat{\mathbf{\Psi}}, \hat{\mathbf{A}}$

Initialize $\underline{\mathbf{S}}, \boldsymbol{\Psi}, \mathbf{A}$ and choose $\lambda_{S}, \lambda_{\Psi}$ and $\lambda_{A} \geq 0$;

while ANLS termination criterion is not satisfied do

$$
\begin{aligned}
& \underline{\mathbf{S}} \leftarrow \underset{\mathbf{S}}{\arg \min } \mathcal{J}(\mathbf{A}, \underline{\mathbf{S}}, \mathbf{\Psi}) ; \\
& \mathbf{\Psi} \leftarrow \underset{\mathbf{S}}{\arg \min } \mathcal{J}(\mathbf{A}, \underline{\mathbf{S}}, \mathbf{\Psi}) ; \\
& \mathbf{A} \leftarrow \underset{\mathbf{\Psi} \geq \mathbf{0}}{\arg \min } \mathcal{J}(\mathbf{A}, \underline{\mathbf{S}}, \mathbf{\Psi}) ;
\end{aligned}
$$

end

Algorithm 1: ANLS scheme to find a local minimum of (8).

\section{A. Optimization w.r.t. $\underline{\mathbf{S}}$}

Rewriting the terms of Eq. (8) depending on $\underline{\mathbf{S}}$, we have to solve:

$$
\underline{\hat{\mathbf{S}}}=\underset{\underline{\mathbf{S}} \geq \mathbf{0}}{\arg \min } \frac{1}{2} \sum_{k=1}^{N}\left(\left\|\mathbf{x}_{k}-\mathbf{S}_{k} \mathbf{a}_{k}\right\|_{F}^{2}+\lambda_{S}\left\|\mathbf{S}_{k}-\mathbf{S}_{0} \boldsymbol{\psi}_{k}\right\|_{F}^{2}\right) .
$$

This problem is completely separable over the $N$ pixels, so its closed form solution may be computed separately for each of them:

$$
\hat{\mathbf{S}}_{k} \leftarrow\left(\mathbf{x}_{k} \mathbf{a}_{k}^{\top}+\lambda_{S} \mathbf{S}_{0} \boldsymbol{\psi}_{k}\right)\left(\mathbf{a}_{k} \mathbf{a}_{k}^{\top}+\lambda_{S} \mathbf{I}_{P}\right)^{-1},
$$

${ }^{1}$ In Eq. (5), there is a scaling ambiguity on the abundances and scaling factors which could be more important without the ASC: in the product of an abundance coefficient in one pixel for one endmember and the corresponding scaling factor is the same if one of the two quantities is multiplied and the other divided by the same constant. 
where $\mathbf{I}_{P}$ is the $P \times P$ identity matrix. The solution $\hat{\mathbf{S}}_{k}$ is then projected onto the nonnegative orthant $\mathbb{R}_{+}^{L \times P}$ by thresholding the negative entries to 0 .

\section{B. Optimization w.r.t. A}

The optimization problem w.r.t. $\mathbf{A}$ is:

$$
\begin{aligned}
& \hat{\mathbf{A}}=\underset{\mathbf{A}}{\arg \min } \frac{1}{2} \sum_{k=1}^{N}\left\|\mathbf{x}_{k}-\mathbf{S}_{k} \mathbf{a}_{k}\right\|_{2}^{2} \\
& +\lambda_{A}\left(\left\|\mathcal{H}_{h}(\mathbf{A})\right\|_{2,1}+\left\|\mathcal{H}_{v}(\mathbf{A})\right\|_{2,1}\right) \\
& +\mathcal{I}_{\mathbb{R}_{+}^{P \times N}}(\mathbf{A})+\boldsymbol{\mu}^{\top}\left(\mathbf{A}^{\top} \mathbb{1}_{P}-\mathbb{1}_{N}\right) .
\end{aligned}
$$

This problem is neither separable w.r.t. the pixels nor to the different endmembers, and it is not differentiable due to the presence of the $\mathcal{L}_{2,1}$ norm. This suggests the use of the popular Alternating Direction Method of Multipliers (ADMM) [33]. This technique will allow us to decompose the hard problem of Eq. (13) into iterations of a sequence of easier subproblems with closed form solutions. In addition, by an appropriate choice of split variables (namely the definition of $\mathbf{B}_{2}$, see below), it will allow us to decouple the optimization in the spectral domain (related to the term in which the endmembers appear) to the optimization in the spatial domain (related to the terms in which the gradient operators appear), in a way similar to [31]. Note that by removing the regularization term $\mathcal{R}(\mathbf{A})$ of Eq.(9), but keeping the ANC and the ASC, the problem becomes the simple FCLSU and can be solved separately in each pixel using for instance the algorithm of [25].

1) Problem formulation: We introduce the splitting variables $\mathbf{B}_{i, i=1, \cdots, 6}$, and express the problem of Eq. (13) as:

$$
\begin{aligned}
\hat{\mathbf{A}} & =\underset{\mathbf{A}}{\arg \min } \frac{1}{2} \sum_{k=1}^{N}\left\|\mathbf{x}_{k}-\mathbf{b}_{1 k}\right\|_{2}^{2} \\
& +\lambda_{A}\left(\left\|\mathbf{B}_{3}\right\|_{2,1}+\left\|\mathbf{B}_{4}\right\|_{2,1}\right) \\
& +\mathcal{I}_{\mathbb{R}_{+}^{P \times N}}\left(\mathbf{B}_{5}\right)+\boldsymbol{\mu}^{\top}\left(\mathbf{A}^{\top} \mathbb{1}_{P}-\mathbb{1}_{N}\right)
\end{aligned}
$$

s.t.

$$
\begin{aligned}
& \mathbf{B}_{1}=\left[\begin{array}{lll}
\mathbf{S}_{1} \mathbf{a}_{1} & \cdots & \mathbf{S}_{N} \mathbf{a}_{N}
\end{array}\right]=\mathcal{S}(\mathbf{A}) \\
& \mathbf{B}_{2}=\mathbf{A} \\
& \mathbf{B}_{3}=\mathcal{H}_{h}\left(\mathbf{B}_{2}\right) \\
& \mathbf{B}_{4}=\mathcal{H}_{v}\left(\mathbf{B}_{2}\right) \\
& \mathbf{B}_{5}=\mathbf{A}
\end{aligned}
$$

where $\mathbf{b}_{i k}$ denotes the $k^{\text {th }}$ column of $\mathbf{B}_{i}$, and $\mathcal{S}$ is the linear operator associated to the definition of $\mathbf{B}_{1}$. Now the optimization problem in Eq. (14) can be expressed in the framework of the ADMM. To do so, we have to rewrite the problem of Eq.(14) in the following form:

$$
\{\hat{\mathbf{u}}, \hat{\mathbf{v}}\}=\underset{\mathbf{u}, \mathbf{v}}{\arg \min } g(\mathbf{v}) \quad \text { s.t. } \quad \boldsymbol{\Gamma} \mathbf{u}+\boldsymbol{\Lambda} \mathbf{v}=\mathbf{0},
$$

where $\mathbf{u}$ and $\mathbf{v}$ are vector variables such that:

$$
\mathbf{u}=\mathbf{a}=\operatorname{vec}(\mathbf{A}) \quad \text { and } \quad \mathbf{v}=\left[\begin{array}{c}
\mathbf{b}_{1} \\
\mathbf{b}_{2} \\
\mathbf{b}_{3} \\
\mathbf{b}_{4} \\
\mathbf{b}_{5}
\end{array}\right]
$$

where $\mathbf{a}=\operatorname{vec}(\mathbf{A})$ is the vectorized version of $\mathbf{A}$. The function $g$ is a closed proper convex function defined as:

$$
\begin{aligned}
g(\mathbf{v}) & =\frac{1}{2}\left\|\mathbf{x}-\mathbf{b}_{1}\right\|_{2}^{2}+\lambda_{A}\left(\left\|\operatorname{vec}^{-1}\left(\mathbf{b}_{3}\right)\right\|_{2,1}\right. \\
& \left.+\left\|\operatorname{vec}^{-1}\left(\mathbf{b}_{4}\right)\right\|_{2,1}\right)+\mathcal{I}_{\mathbb{R}_{+}^{P N}}\left(\mathbf{b}_{5}\right)+\boldsymbol{\mu}^{\top}\left(\mathbf{K a}-\mathbb{1}_{N}\right)
\end{aligned}
$$

Here $\mathbf{x}=\operatorname{vec}(\mathbf{X})$ is the vectorized version of $\mathbf{X}$ (by concatenating its columns), and analogously $\mathbf{b}_{i}=\operatorname{vec}\left(\mathbf{B}_{i}\right)$. $\mathbf{K}$ is the $N \times P N$ matrix of the linear operator summing the entries of a corresponding to the same pixel and putting each of these sums in one entry of a vector. Finally, we have the following definitions for $\boldsymbol{\Gamma}$ and $\boldsymbol{\Lambda}$ :

$$
\boldsymbol{\Lambda}=\left[\begin{array}{cccccc}
-\mathbf{I}_{L N} & \mathbf{0} & \mathbf{0} & \mathbf{0} & \mathbf{0} \\
\mathbf{0} & -\mathbf{I}_{P N} & \mathbf{0} & \mathbf{0} & \mathbf{0} \\
\mathbf{0} & \mathbf{H}_{h} & -\mathbf{I}_{P N} & \mathbf{0} & \mathbf{0} \\
\mathbf{0} & \mathbf{H}_{v} & \mathbf{0} & -\mathbf{I}_{P N} & \mathbf{0} \\
\mathbf{0} & \mathbf{0} & \mathbf{0} & \mathbf{0} & -\mathbf{I}_{P N}
\end{array}\right],
$$

using the fact that in the vector spaces of vectorized matrices of the appropriate sizes, the linear operators $\mathcal{H}_{h}, \mathcal{H}_{v}$ : $\mathbb{R}^{P \times N} \mapsto \mathbb{R}^{P \times N}$ and $\mathcal{S}: \mathbb{R}^{P \times N} \mapsto \mathbb{R}^{L \times N}$ can be described by their matrices (in the canonical bases of the corresponding vector spaces) $\mathbf{H}_{h}, \mathbf{H}_{v} \in \mathbb{R}^{P N \times P N}$ and $\boldsymbol{\Sigma} \in \mathbb{R}^{L N \times P N}$, respectively. Here $\mathbf{I}_{(\cdot)}$ denotes the identity matrix whose size is given in its index.

In this framework, the problem we want to solve falls into the category of those which the ADMM can tackle. We have introduced two equivalent representations of the variables we manipulate: in a matrix form and in a vector form. The matrix form is more compact and often convenient to use, but the vector form is the only one allowing us to express linear operators as matrices. The two are completely equivalent (up to an isomorphism) and during the optimization process, we will use either of them depending on which one is the most convenient in the context.

2) ADMM framework: The ADMM technique consists in expressing the constrained problem defined in Eq. (15) in an unconstrained way using an Augmented Lagrangian (AL), and then minimizing it iteratively and alternatively for each of the variables introduced, including the Lagrange Multipliers appearing in the AL (the so-called dual update). $\rho$ is the barrier parameter weighting the AL terms, and the (scaled) Lagrange mutlipliers are denoted by $\mathbf{d}$ in a vector form, possibly indexed with the pixels (and possibly with the index of the appropriate split variable), or $\mathbf{D}$ in a matrix form. The procedure is summed up in Algorithm 2.

\section{Data: X, $\underline{\mathrm{S}}$}

Result: $\hat{\mathbf{A}}=\operatorname{vec}^{-1}(\mathbf{u})$

Choose $\rho \geq 0$ and initialize $\mathbf{u}, \boldsymbol{\mu}, \mathbf{v}$ and $\mathbf{d}$;

while ADMM termination criterion is not satisfied do

$\mathbf{u}, \boldsymbol{\mu} \leftarrow \arg \min \mathcal{L}(\mathbf{u}, \boldsymbol{\mu}, \mathbf{v}, \mathbf{d}) ;$

$\mathbf{v} \leftarrow \arg \min \mathcal{L}(\mathbf{u}, \boldsymbol{\mu}, \mathbf{v}, \mathbf{d}) ;$

$$
\mathbf{d} \leftarrow \mathbf{d}-\mathbf{v} \mathbf{u}-\Lambda \mathbf{v}
$$

Algorithm 2: ADMM process to solve problem (15).

The augmented Lagrangian for our problem, to be minimized w.r.t. $\mathbf{u}, \boldsymbol{\mu}, \mathbf{v}$ and $\mathbf{d}$ is: 


$$
\begin{array}{r}
\mathcal{L}(\mathbf{u}, \boldsymbol{\mu}, \mathbf{v}, \mathbf{d})=f(\mathbf{u})+g(\mathbf{v})+\frac{\rho}{2}\left(\|\mathbf{\Gamma} \mathbf{u}+\boldsymbol{\Lambda} \mathbf{v}-\mathbf{d}\|_{2}^{2}-\|\mathbf{d}\|_{2}^{2}\right) \\
=\frac{1}{2}\left\|\mathbf{x}-\mathbf{b}_{1}\right\|_{2}^{2}+\lambda_{A}\left(\left\|\operatorname{vec}^{-1}\left(\mathbf{b}_{2}\right)\right\|_{2,1}+\left\|\operatorname{vec}^{-1}\left(\mathbf{b}_{3}\right)\right\|_{2,1}\right) \\
+\mathcal{I}_{\mathbb{R}_{+}^{P N}}\left(\mathbf{b}_{4}\right)+\boldsymbol{\mu}^{\top}\left(\mathbf{K u}-\mathbb{1}_{N}\right)+\frac{\rho}{2}\left\|\mathbf{\Sigma} \mathbf{u}-\mathbf{b}_{1}-\mathbf{d}_{1}\right\|_{2}^{2} \\
+\frac{\rho}{2}\left\|\mathbf{u}-\mathbf{b}_{2}-\mathbf{d}_{2}\right\|_{2}^{2}+\frac{\rho}{2}\left\|\mathbf{H}_{h} \mathbf{b}_{2}-\mathbf{b}_{3}-\mathbf{d}_{3}\right\|_{2}^{2} \\
+\frac{\rho}{2}\left\|\mathbf{H}_{v} \mathbf{b}_{2}-\mathbf{b}_{4}-\mathbf{d}_{4}\right\|_{2}^{2}+\frac{\rho}{2}\left\|\mathbf{u}-\mathbf{b}_{5}-\mathbf{d}_{5}\right\|_{2}^{2}-\frac{\rho}{2}\|\mathbf{d}\|_{2}^{2}
\end{array}
$$

The full optimization procedure for the ADMM is described in detail in a supplementary material file provided by the authors.

\section{Optimization w.r.t. $\Psi$}

Rewriting the terms of the criterion (8) depending only on $\Psi$, we get:

$$
\begin{aligned}
\hat{\mathbf{\Psi}} & =\underset{\boldsymbol{\Psi}}{\arg \min } \frac{\lambda_{S}}{2} \sum_{k=1}^{N}\left\|\mathbf{S}_{k}-\mathbf{S}_{0} \boldsymbol{\psi}_{k}\right\|_{F}^{2} \\
& +\frac{\lambda_{\Psi}}{2}\left(\left\|\mathcal{H}_{h}(\mathbf{\Psi})\right\|_{F}^{2}+\left\|\mathcal{H}_{v}(\boldsymbol{\Psi})\right\|_{F}^{2}\right) .
\end{aligned}
$$

We can see $\underline{\mathbf{S}}=\left\{\mathbf{S}_{k}\right\}$ as an $L \times N \times P$ cube, with $P$ $L \times N$ slices corresponding to the source matrices in every pixel. Using this description, we can rewrite Eq. (20) in a way that is separable w.r.t. the different materials:

$$
\begin{gathered}
\hat{\boldsymbol{\Psi}}=\underset{\boldsymbol{\Psi}}{\arg \min } \frac{\lambda_{S}}{2} \sum_{p=1}^{P}\left(\left\|\mathbf{S}^{p}-\mathbf{s}_{0}^{p}\left(\boldsymbol{\psi}^{p}\right)^{\top}\right\|_{F}^{2}\right. \\
\left.+\frac{\lambda_{\Psi}}{2}\left(\left\|\mathcal{H}_{h}\left(\boldsymbol{\psi}^{p}\right)\right\|_{2}^{2}+\left\|\mathcal{H}_{v}\left(\boldsymbol{\psi}^{p}\right)\right\|_{2}^{2}\right)\right),
\end{gathered}
$$

where $\mathbf{S}^{p}$ is a $L \times N$ slice of the cube, $\mathbf{s}_{0}^{p}$ is a column of $\mathbf{S}_{0}$ (representing one reference endmember). $\psi^{p}$ is the $p^{\text {th }}$ column of $\boldsymbol{\Psi}$ (a $N \times 1$ vector containing the scaling factors for all the pixels for one material). The update for $\boldsymbol{\psi}^{p}$ is:

$\boldsymbol{\psi}^{p} \leftarrow\left(\left(\lambda_{S} \mathbf{s}_{0}^{p^{\top}} \mathbf{s}_{0}^{p}\right) \mathbf{I}_{N}+\lambda_{\psi}\left(\mathbf{H}_{h}^{\top} \mathbf{H}_{h}+\mathbf{H}_{v}^{\top} \mathbf{H}_{v}\right)\right)^{-1}\left(\lambda_{S} \mathbf{S}^{p^{\top}} \mathbf{s}_{0}^{p}\right)$.

The $N \times N$ matrix inversion is intractable as such in most cases, but as the matrix is circulant the update can be very efficiently computed in the Fourier domain (assuming periodic boundaries for each $\psi^{p}$ image) by:

$\psi^{p} \leftarrow \mathcal{F}^{-1}\left(\frac{\mathcal{F}\left(\lambda_{S} \mathbf{S}^{p^{\top}} \mathbf{s}_{0}^{p}\right)}{\left(\lambda_{S} \mathbf{s}_{0}^{p \top} \mathbf{s}_{0}^{p}\right) \mathbb{1}_{m \times n}+\lambda_{\Psi}\left(\left|\mathcal{F}\left(\mathbf{h}_{h}\right)\right|^{2}+\left|\mathcal{F}\left(\mathbf{h}_{b}\right)\right|^{2}\right)}\right)$

where $\mathcal{F}$ and $\mathcal{F}^{-1}$ denote the Discrete 2D Fourier Transform and its inverse, $m$ and $n$ are the spatial dimensions of the image, such that $m \times n=N$, and $\mathbf{h}_{h}$ and $\mathbf{h}_{v}$ are convolution masks for the gradient operators (see the supplementary material for more details).

\section{EXPERIMENTS ON SYNTHETIC DATA}

In this section, we present the experiments performed on two types of synthetic datasets to validate the proposed approach. In both cases, we will compare the proposed approach with the classical FCLSU and CLSU, but also with the bundles approach used with SUnSAL, and the FDN algorithm. Finally, we will also compare the proposed approach with S-CLSU, which follows a particular case of the ELMM. Since the ELMM algorithm makes use of spatial regularization, for a fairer comparison, we also include the results for modified versions of the competing algorithms, in which a TV on the abundances is enforced, using the SUnSAL-TV code of [31]. We only added a termination criterion similar to the one used for the proposed approach, that is when the relative variation (in norm) of the abundance matrix between two consecutive iterations goes below $\epsilon_{A}=10^{-3}$. Finally, we also compare the results of the proposed approach with the ones of the Perturbed Linear Mixing Model (PLMM) algorithm [21], which is an algorithm specifically designed to tackle the spectral variability issue. It models spectral variability in each pixel as an additive perturbation of reference endmembers, and hence is able to estimate the variability for each material and each pixel by computing the norm of the perturbation term.

The proposed approach with both regularizations enforced is denoted by ELMM-A $\psi$. For the ELMM algorithm, the three tolerances $\epsilon_{A}=\epsilon_{S}=\epsilon_{\Psi}$ were set to $10^{-3}$. When no spatial regularization is performed on the abundances or the scaling factors, we simply refer to the algorithm as ELMM. The running times of the different algorithms were measured on a computer using an Intel $®$ Core $^{\mathrm{TM}}$ i7-4770 CPU @ 3.40GHz (except for the PLMM).

\section{A. First scenario}

1) Data: The first dataset on which we tested the proposed approach was designed to follow the ELMM with some perturbations. The idea is to build a dataset which is halfway between a toy example and a realisitic simulation, in order to compare easily the different algorithms and to explain the properties and particularities of the proposed method. We randomly chose five reference endmembers corresponding to the signatures of minerals from the United States Geological Survey (USGS) spectral library, comprising 224 spectral bands in the visible and near-IR. They are shown in Fig. 3. The $200 \times 200$ abundance maps used were generated using Gaussian Fields, and were designed to comply with the ASC. Note that these abundance maps comprise only one pure pixel for each material, and around 5\% of the pixels have an abundance coefficient superior to 0.9 for one material. We also generated spectral variability maps for each endmember using mixtures of Gaussians. The true abundances are shown in Fig. 4 and the true scaling factors are shown in Fig. 5. Then, the dataset was generated as follows: the pixel-dependent endmember instances were generated by multiplying the references by the corresponding spectral variability scaling factors (the achievable values are chosen so that no reflectance value becomes higher than 1, and so that the scaling factors range from 0.75 to 1.25 ), and a $25 \mathrm{~dB}$ white Gaussian noise was added to these endmembers. Then for each pixel, the mixture was performed 


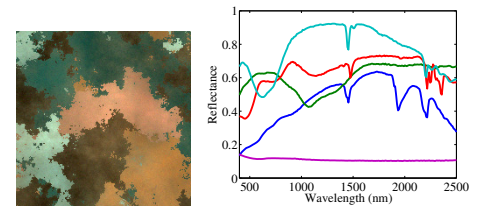

Fig. 3. A false color representation of the first synthetic dataset (left) and the endmembers used for the simulation.

using the LMM, and finally we added a $25 \mathrm{~dB}$ white Gaussian noise to the generated pixels. The process then yielded a $200 \times 200 \times 224$ simulated hyperspectral image. A false color representation of the data can be seen in Fig. 3.

2) Experimental setup: For each algorithm, the used EEA was the Vertex Component Analysis (VCA), and the same set of 5 endmembers was used for all the algorithms which do not require a bundle. For the bundles, we extracted 5 endmembers instances on 50 randomly chosen susbets (without replacement) of the image whose number of pixels was $2 \%$ of this of the whole image. The clustering into bundles was performed with the k-means algorithm, with the spectral angle as a similarity measure (it is insensitive to scalings and hence adapted to the problem). The different regularization parameters used for the tested algorithms were set empirically so as to get the best performance possible, and are gathered in the supplementary material file.

The initialization of the proposed algorithm is important since the optimization problem we tackle is not convex. We chose to initialize the algorithm using the abundances of SCLSU, every scaling factor set to one, and the five reference endmembers as well as the initial sources in each pixel were the ones extracted using VCA.

In order to assess the performance of the algorithms, we define the abundance overall Root Mean Square Error (aRMSE) as:

$$
a R M S E=\frac{1}{N} \sum_{k=1}^{N} \sqrt{\frac{1}{P} \sum_{p=1}^{P}\left(a_{p k_{t r u e}}-\hat{a}_{p k}\right)^{2}},
$$

and the overall source RMSE (sRMSE) as:

$$
s R M S E=\frac{1}{N} \sum_{k=1}^{N} \sqrt{\frac{1}{L P}\left\|\mathbf{S}_{k_{\text {true }}}-\hat{\mathbf{S}}_{k}\right\|_{F}^{2}} .
$$

This metric allows us to measure indirectly how well the spectral variability is recovered by comparing the true sources to the ones extracted by S-CLSU and the proposed approach. A direct comparison using the scaling factors would have been harder to perform since the extracted reference endmembers can differ from the ones actually used to generate the data, and because of the additive perturbation added to the scaled signatures. Finally, we also define two measures to assess the quality of the reconstruction of the data from the estimated parameters and the mixing model used by an algorithm:

$$
x R M S E=\frac{1}{N} \sum_{k=1}^{N} \sqrt{\frac{1}{L} \sum_{l=1}^{L}\left(x_{l k_{\text {true }}}-\hat{x}_{l k}\right)^{2}},
$$

This is the usual average RMSE on the data reconstruction. We finally define the average Spectral Angle Mapper (SAM) between the actual and reconstructed data:

$$
x S A M=\frac{1}{N} \sum_{k=1}^{N} \arccos \left(\frac{\mathbf{x}_{k_{\text {true }}} \cdot \hat{\mathbf{x}}_{k}}{\left\|\mathbf{x}_{k_{\text {true }}}\right\|\left\|\hat{\mathbf{x}}_{k}\right\|}\right),
$$

where $\cdot$ is the dot product.

3) Results: The quantitative results of this experiment are shown in Table I. A visual representation of the extracted abundances for most algorithms is shown in Fig. 4, while the scaling factors extracted by S-CLSU and the proposed approach, as well as the variability estimation from the PLMM are shown in Fig. 5.

From the results, we can see that as expected, FCLSU performs rather poorly in a scenario where spectral variability comes into play. Sinces the endmember signatures are constant throughout the image, a scaled endmember can be easily mistaken for another, for instance with the endmembers depicted in cyan and red in Fig. 3. The bundles approach is able to obtain better results, provided the bundles are balanced and representative of the spectral variability present in the scene. This is not always guaranteed and can lead to erroneous estimations. In these experiments, we show the best result for this approach out of 15 runs. The bundles allow several instances of each endmember to be considered. The sparsity enforced by SUnSAL helps reducing the number of active endmembers per pixel but there can still remain several endmembers of the same endmember class contributing to one pixel value. The FDN approach is allowed to reduce the dimensionality of the dataset such that the impact of spectral variability is lowered.

The results from the PLMM are in this case comparable to those of the algorithms which use bundles. The main advantage of this algorithm is that it is able to estimate spectral variability maps by computing the power of the additive perturbation term in each pixel, although in this case the abundances are relatively close to the ones of FCLSU. We can see that globally, the algorithm is able to roughly identify the regions where most of the spectral variability occurs (corresponding to red or dark blue pixels in the true scaling factors maps when the true scaling factor is significantly above or below 1 , respectively). The main drawback is that the algorithm is not able to extrapolate the information in high abundance pixels to neighboring lower abundance pixels, on which spectral variability is harder to estimate without considering spatial information.

The CLSU algorithm performs better than FCLSU and all the previously mentioned approaches, since dropping the ASC allows to look for the abundances in a cone and not in a simplex. However, the quantity estimated in each pixel by CLSU actually absorbs spectral variability into the abundances. SCLSU performs much better. It is a very simple approach to adress spectral variability which is well suited in simple cases. The cases in which it performs best are those in which there are few materials in the image, or/and when the scaling factors are either correlated along different materials, or on the contrary if only one material per pixel varies significantly from its reference signature. This approach is also sensitive to deviations from the ELMM such as a noisy perturbation on the scaled signatures. 
The spatial regularization on the abundances logically improves the results for all the algorithms based on CLSU, but it cannot improve the results for the bundle approaches. Indeed, one drawback of the SUnSAL-TV algorithm is that it cannot enforce the ASC, since it is not compatible with the $\mathcal{L}_{1}$ norm minimization (the ASC forces the $\mathcal{L}_{1}$ norm of the abundance vectors to be constant). What is more, the noisiness of the abundance maps obtained with the bundles is not ideally corrected by the TV, which tends to aggregate "noisy" areas of the abundance maps into patches. However, the combination of the spatial regularizations on the abundances and scaling factors, coupled with the explicit scaling factor estimation is able to improve the results significantly.

Indeed, the proposed approach is much more robust to noise on the measured data as well as on the signatures thanks to both spatial regularizations. Indeed, the spatial regularization allows to estimate precisely the spatially correlated abundances, getting rid of the noise and the uncertainty which affects S-CLSU when two endmember variations of two different materials share a common global shape, and can look quite similar after appropriate scalings. The spatial coherency of the abundances and the scaling factors allows to recover the parameters more precisely. Besides, the explicit computation of a different scaling factor for each pixel and material allows to obtain smoother and separated variability maps, which also makes the proposed algorithm much stronger in terms of interpretability of its results. Of course, it is only possible to recover accurately the scaling factors when the abundance contribution of the corresponding material is high enough, or otherwise when the spatial information allows to extrapolate from higher abundance areas. If those two conditions are missing, only the abundance is recovered with precision, while the associated scaling factor tends to be close to one (its initial value) as the abundance decreases. This phenomenon can be interpreted geometrically, with the diagram of Fig. 2 in mind. Let us suppose that there are three endmembers in the scene. If in a given pixel, the abundance of one material is low, then a different scaling factor for this material will change the orientation of the simplex related to this pixel, but the edge of the simplex linking the other two (scaled) endmembers will not change, and thus the abundance coefficients for the other two materials will not change much either. In the end, we can say that the proposed approach does not require pure pixels to extract the spectral variability of a material efficiently, but only a significant abundance contribution of this material in the considered pixel, or in the neighboring area.

From a quantitative point of view, we can see that the proposed approach obtains the best results in terms of abundance estimation, as well as spectral variability recovery. An explicit spectral variability map can be only recovered for the S-CLSU, PLMM and ELMM algorithms, since only those algorithms estimate pixel-dependent endmembers, and thus enable us to compute the sRMSE values.

It is interesting to note that both spatial regularizations improve the results on their own w.r.t. to the simple ELMM case (the spatial regularization on the abundances (resp. scaling factors) improves the abundance (resp. scaling factors) estimation), but the combination of both improves the results further both for abundance and spectral variability estimation, since a better estimation of the scaling factors allows in turn a better abundance estimation, and vice versa. The regularizations also improve the conditioning of the problem, and help to solve the ambiguity between abundances and scaling factors. The running time of the proposed algorithm is more important than all others (except the PLMM), but the approach is relatively fast thanks to the favorable initialization chosen.It allows to achieve a good local miminum of the objective function while limiting the number of iterations necessary to reach it with a reasonable precision. However, we can note that S-CLSU performed with SUnSAL-TV (which is based on the ADMM technique) is faster than the usual nonnegative least squares, even with the spatial regularization (at least for $\epsilon_{\Psi}=10^{-3}$ ).

We also compared the reconstruction errors of the different algorithms, in terms of Root Mean Squared Error and in terms of Spectral Angle. These measures are indirect, in the sense that they only show how the mixing model used fits the data, though it is possible to achieve excellent reconstruction errors with a poor abundance and/or spectral variability retrieval. Conversely, accurate parameter estimation entails a good reconstruction if the model is suited to the data. For instance, we see that the bundle-based approaches fit the data worse than FCLSU, although the abundance estimation is significantly improved. We also see that for this data, all the models based on unmixing the data in a cone spanned by three endmembers achieve better reconstruction errors (as well as abundance estimation). For CLSU, S-CLSU, and the ELMMbased algorithms, the reconstruction error is similar, but there are still important differences in the accuracy of the estimation of the parameters. For the case of CLSU, only a simple scaling has a positive effect on the abundance estimation, while the reconstruction errors are of course the same in this case. In the supplementary material file are also shown a sensitivity analysis of the algorithm to the regularization parameters and an analysis of the effect of smoothing the scaling factors.

\section{B. Second scenario}

1) Data: The second dataset we used was generated in order to mimick the spectral variability induced by changing illumination conditions and topography along the scene, using a physical model introduced by Hapke [29]. This model allows one to access the reflectance value of a material for one wavelength, knowing the corresponding single scattering albedo, the photometric parameters of the material, as well as the incidence, emergence, and azimuth angles during the acquisition. We refer the reader to [27] and references therein for more details. For the simulations, we selected 3 endmembers consisting in 16 wavelengths reflectance measurements for materials commonly found on small bodies of the Solar System (basalt, palagonite and tephra), and whose geometry for the acquisition, as well as their photometric parameters are known [34]. We show these reflectance spectral signatures, acquired at nadir with an incidence angle of $30^{\circ}$, in Fig 6. Note that palagonite and tephra are spectrally very close (the spectral angle between the two materials is 10 degrees), making the problem harder since the nonlinearities of the Hapke model will have more influence on the abundances for correlated endmembers. From these data, we recovered 


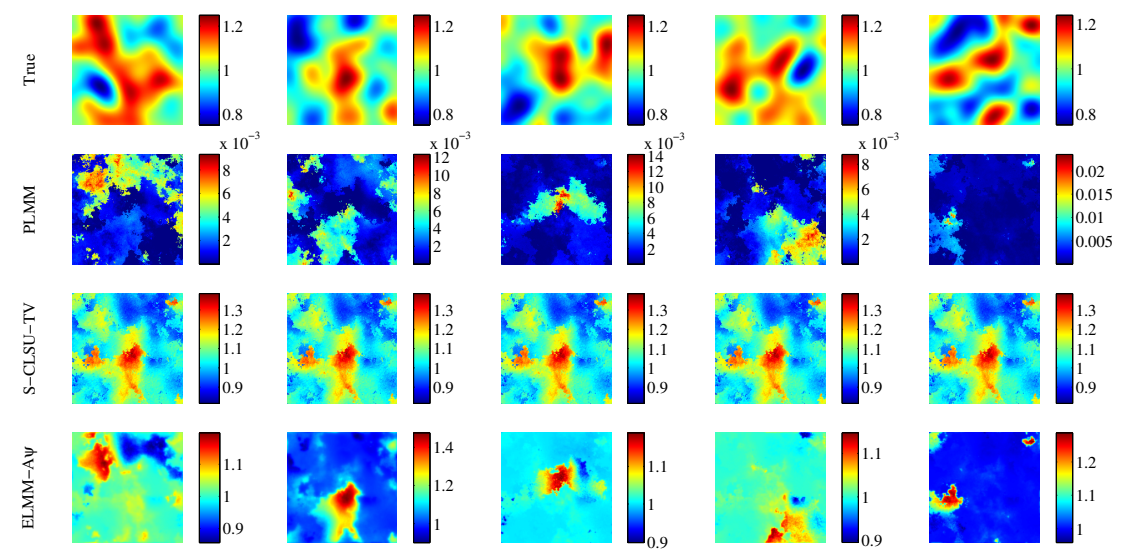

Fig. 5. The scaling factors estimated by S-CLSU (third row) and by the proposed approach (bottom row), compared to the true ones, and to the power of the variability estimated by the PLMM algorithm (second row) for the first synthetic dataset.

\begin{tabular}{|c|c|c|c|c|c|c|c|c|c|c|c|c|}
\hline Algorithm & FCLSU & \multicolumn{2}{|c|}{ BUNDLES + SUnSAL } & \multicolumn{2}{c|}{ BUNDLES + FDN } & \multicolumn{2}{|c|}{ CLSU } & PLMM & \multicolumn{2}{c|}{ S-CLSU } & ELMM & ELMM-A $\psi$ \\
\hline SR on abundances & No & No & Yes & No & Yes & No & Yes & No & Yes & No & No & Yes \\
\hline aRMSE & 0.0629 & 0.0490 & 0.0504 & 0.0407 & 0.0575 & 0.0432 & 0.0413 & 0.0886 & 0.0276 & 0.0269 & 0.0344 & 0.0199 \\
\hline sRMSE & $\times$ & $\times$ & $\times$ & $\times$ & $\times$ & $\times$ & $\times$ & 0.0614 & 0.0548 & 0.0545 & 0.0449 & 0.0439 \\
\hline xRMSE & 0.0119 & 0.0213 & 0.0366 & 0.0331 & 0.1391 & 0.0085 & 0.0100 & 0.0136 & 0.0085 & 0.0100 & 0.0090 & 0.0088 \\
\hline xSAM (degrees) & 1.4960 & 1.8752 & 2.1304 & 1.8716 & 7.9886 & 1.2268 & 1.2504 & 1.9231 & 1.2268 & 1.2504 & 1.3002 & 1.2433 \\
\hline Running Time (s) & 14 & 26 & 131 & 18 & 8 & 16 & 6 & 311 & 17 & 7 & 366 & 399 \\
\hline
\end{tabular}

TABLE I

QUANTITATIVE RESULTS FOR THE FIRST SYNTHETIC DATASET. THE BEST VALUES IN EACH LINE IS SHOWN IN RED, AND THE SECOND BEST ONE IS SHOWN IN BLUE.

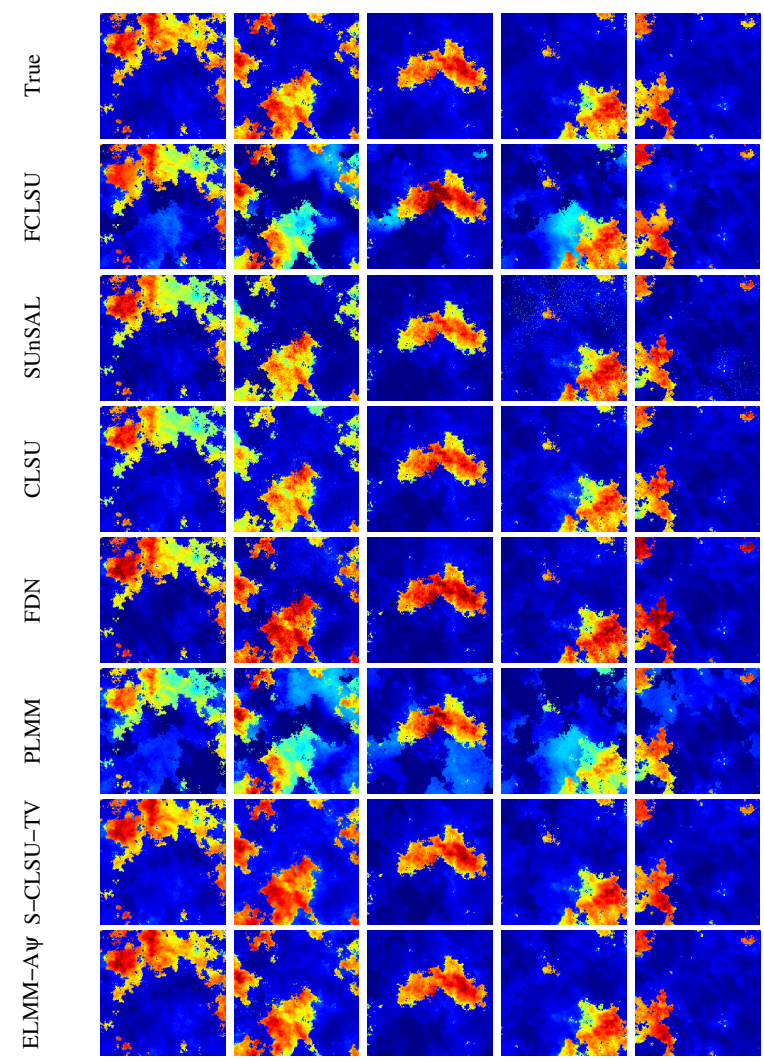

Fig. 4. The abundances estimated by all algorithms (each column corresponds to one endmember) for the first synthetic dataset, compared to the true ones (first row). the single scattering albedo spectra of these materials by inverting the Hapke model [35]. Single scattering albedo is completely characteristic to a material, and unlike reflectance, which is the physical quantity we work with, it depends neither on the geometry of the scene nor on the illumination conditions [27]. Separately, a simulated smooth $200 \times 200$ Digital Terrain Model (DTM) was synthesized, assuming a spatial resolution of $1 \mathrm{~m}$. This DTM simulates a hilly region and is shown in Fig. 6. From this model and the definition of the position of the sun and the sensor w.r.t. the scene (sun making an angle of $18^{\circ}$ with the flat part of the DTM and sensor at nadir), we derived the acquisition angles associated to each pixel. They depend on the position of the sun and sensor, but also on the orientation of the tangent plane to the surface at each location, which itself depends on the topography of the scene. We show the computed angles in Fig.7. Plugging these angles, the single scattering albedos and the photometric parameters into the Hapke model, we simulated the various instances of the reflectance endmembers along the scene. Then we mixed these endmember variants in each pixel using the LMM, using abundances generated in a similar way to the previous section (with the same pixel purity characteristics), providing a $200 \times 200 \times 16$ image. A false color composition, and a representation of the dataset (in blue) and the endmembers generated by the Hapke model (in red) using the first three components of a Principal Component Analysis (PCA) are shown in Fig.8. This representation shows that there are strictly speaking very few (one per material, actually) pure pixels in the image. This figure confirms that palagonite and tephra are spectrally close, when we look at the scale of the second principal component. Furthermore, we 


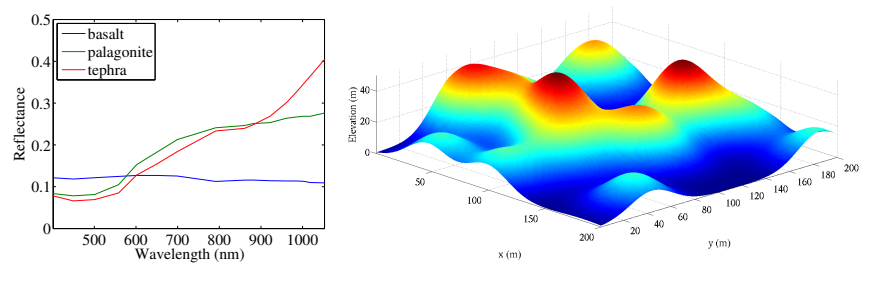

Fig. 6. The reflectance endmembers (left) and the Digital Terrain Model used for the second synthetic dataset (right).
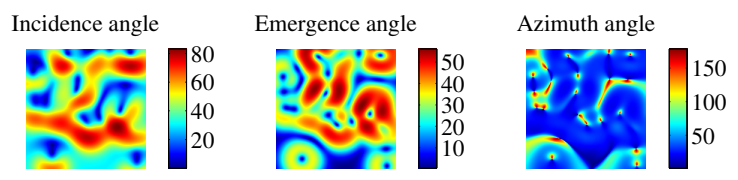

Fig. 7. The incidence, emergence and azimuth angles computed from the DTM.

can see that the different materials are not equally affected by spectral variability. The endmembers corresponding to basalt are less affected by the nonlinearities of the Hapke model, which have a stronger influence on high albedo materials, whereas the spectrum of basalt is very flat and low. Hence the shape of the variability is almost a straight line. For the other two materials, the manifold of the endmember variants is, however, more complex.

2) Experimental setup: The setup for this dataset is rather similar to this of the first synthetic dataset, with a few notable differences. The regularization parameters for all algorithms are given in the supplementary material file. For this data, we chose to set the mean of each extracted endmember bundle as the reference endmembers. The idea is to obtain representative endmembers to increase the robustness of the algorithm. For a fair comparison, we chose the same set of endmembers for FCLSU, CLSU and S-CLSU. The initial abundances used are those of S-CLSU and the initial scaling factors ar either set to one (approach denoted by ELMM-A $\psi$ ), or taken from the results of S-CLSU as well (approach denoted by ELMM-A $\psi$ C).

3) Results: The quantitative results of this second experiment are shown in Table II. A visual representation of the extracted abundances for most algorithms is shown in Fig. 9, while the scaling factors extracted by S-CLSU and the proposed approach (ELMM-A $\psi-\mathrm{C}$ ) are shown in Fig. 10. We also show the endmembers estimated by the proposed approach, compared the true ones using a PCA in Fig 11.
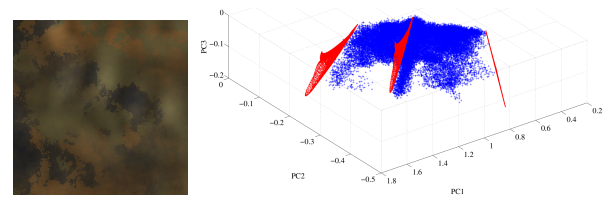

Fig. 8. A false color representation of the second synthetic dataset. Data cloud (blue) and the endmember variants generated by the Hapke model (red) shown using the first three components of a PCA.

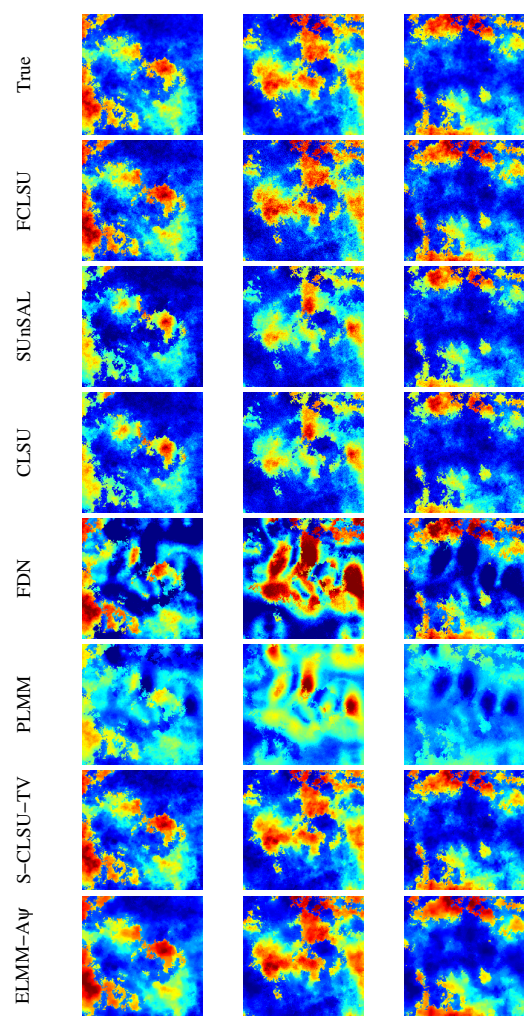

Fig. 9. The abundances estimated by all algorithms for the second synthetic dataset, compared to the true ones.

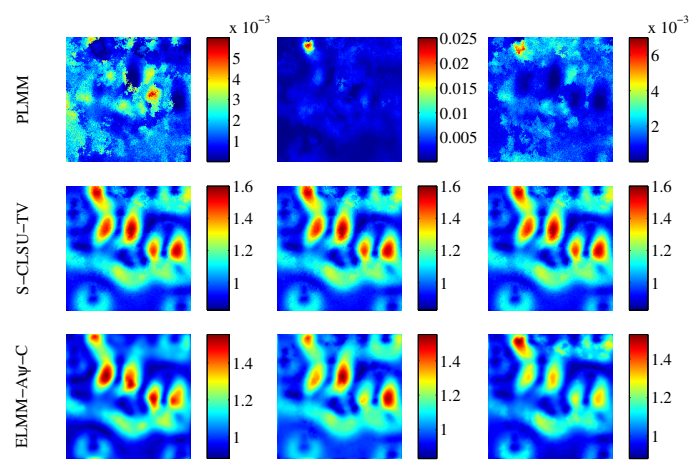

Fig. 10. Magnitude of the PLMM variability term (top row), the scaling factors estimated by S-CLSU (middle row) and by the proposed approach (bottom row) for the second synthetic dataset.

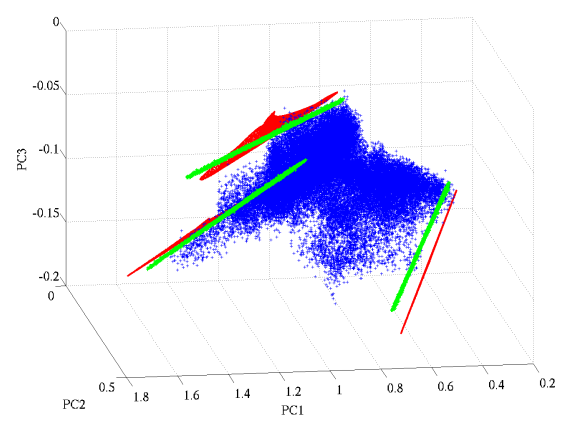

Fig. 11. The second simulated dataset (blue), the endmember variants generated by the Hapke model (red) and the sources estimated by the proposed algorithm (green), shown using the first three components of a PCA. 


\begin{tabular}{|c|c|c|c|c|c|c|c|c|c|c|c|c|}
\hline Algorithm & FCLSU & \multicolumn{1}{|c|}{ BUNDLES + SUnSAL } & \multicolumn{2}{c|}{ BUNDLES + FDN } & \multicolumn{2}{|c|}{ CLSU } & \multicolumn{2}{c|}{ PLMM } & \multicolumn{2}{c|}{ S-CLSU } & ELMM-A $\psi$ & ELMM-A $\psi$-C \\
\hline TV on abundances & No & No & Yes & No & Yes & No & Yes & No & No & Yes & Yes & Yes \\
\hline aRMSE & 0.133 & 0.0860 & 0.0498 & 0.1330 & 0.0681 & 0.0676 & 0.0601 & 0.1445 & 0.0398 & 0.0300 & 0.0297 & 0.0290 \\
\hline sRMSE & $\times$ & $\times$ & $\times$ & $\times$ & $\times$ & $\times$ & $\times$ & 0.0814 & 0.0139 & 0.0135 & 0.0164 & 0.0129 \\
\hline xRMSE & 0.0131 & 0.0105 & 0.0049 & 0.0716 & 0.0577 & 0.0041 & 0.0045 & 0.0052 & 0.0041 & 0.0045 & 0.0048 & 0.0049 \\
\hline xSAM (degrees) & 2.0209 & 1.0906 & 1.1317 & 8.7639 & 7.9573 & 1.0882 & 1.1270 & 1.2035 & 1.0882 & 1.1270 & 1.2834 & 1.1688 \\
\hline Running Time (s) & 10 & 15 & 152 & 10 & 7 & 11 & 6 & 235 & 12 & 7 & 234 & 248 \\
\hline
\end{tabular}

TABLE II

QUANTITATIVE RESULTS FOR THE SECOND SYNTHETIC DATASET. THE BEST VALUES IN EACH LINE IS SHOWN IN RED, AND THE SECOND BEST ONE IS SHOWN IN BLUE.

From Fig. 9 and Table II. we can see that for FCLSU, CLSU, and the combination of the bundles and SUnSAL, the results are similar to those of Sec. IV-A3. FCLSU gets poor results since it does not take spectral variability into account. CLSU obtains better results, as it does not really estimate the abundances, but their products with the scaling factors. SUnSAL is able to partly explain endmember variability, but the resulting abundance maps are noisy, and the performance is limited by the bundle extraction. The FDN approach only provides slightly better results than FCLSU in this case (we kept the best result over 15 bundle extractions). This might be because the projection performed by the FDN approach suffers from outliers in the bundles. SUnSAL obtains better results with the same bundles because the sparsity constraint helps discarding the outlier endmembers. The S-CLSU approach obtains better results, and is able to recover an average spectral variability map. In this case, its performance in terms of sRMSE (but not aRMSE) is better than the proposed approach initialized with the abundances S-CLSU obtains and the scaling factors set to one. The reason S-CLSU obtains satisfactory results is because the spectral variability in the different materials have the same cause, and hence the scaling factors for each material are correlated. However, the ELMM$\mathrm{A} \psi$ initialization also gets a worse endmember estimation result because it cannot estimate the scaling factor of a material whose abundance is too low. As explained in Sec. IV-A3, the change in orientation of the simplex due to the scaling factor associated to a low abundance material has little impact on the remaining abundance coefficients. As S-CLSU is only able to estimate one scaling factor for each endmember, if we assume it applies to all endmembers, the error committed is less important. A spatial regularization on the abundances if beneficial to the performance of the various algorithms, especially for the bundles approach with SUnSAL, as well as S-CLSU. The PLMM obtains poor results here because its abundances are similar to those of FCLSU, which means the algorithm is probably stuck in a poor local minimum. The proposed approach, initialized with the abundances and scaling factors of S-CLSU is still able to improve the results thanks to the regularizations, which accommodate the noise, and especially the nonlinearities of the Hapke model, as can be seen in Fig. 11. In this figure, we represent the data cloud (in blue) and the endmembers generated by the Hapke model (in red) using the first 3 components of a PCA. The endmembers estimated in every pixel by the proposed algorithm are shown in green. The extracted endmembers are allowed to deviate from the ELMM (as it is defined in Eq. (5) ), and the endmembers are extracted on a thickened line. This flexibility

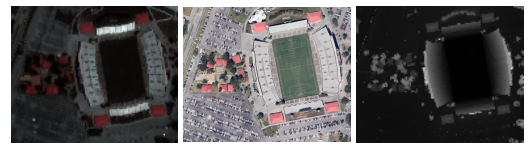

Fig. 12. A RGB representation of the hyperspectral dataset (left). High spatial resolution color image acquired over the same area at a different time (middle). Associated Lidar data (right). Black corresponds to $9.6 \mathrm{~m}$ and white corresponds to $46.2 \mathrm{~m}$.

allows to approximate the endmember manifolds generated by the Hapke Model better than S-CLSU does. Only the basalt endmembers are more or less situated on a straight line, because this material has a lower albedo than the other two, and is then less affected by the illumination changes over the scene. It is also interesting to note that, as could be expected, the scaling factors extracted by S-CLSU or the proposed approach are very correlated to the DTM, and even more to the spatial maps of the incidence and emergence angles, shown in Fig. 7. However, the proposed approach is able to drift away from the the model of Eq. (5) and is more robust than SCLSU here thanks to both spatial regularizations (even when the S-CLSU benefits from an additional spatial regularization on the abundances). In this case, the freedom of the sources to evolve around the straight lines cause a slight increase in the spectral angle between the image and its reconstruction. This could also be because the spatial regularizations denoise the abundance and scaling factor maps, leading to a smoother reconstructed image than the noisy data. These results confirms the potential of the ELMM to deal with illumination and topography induced spectral variability.

\section{EXPERIMENTS ON REAL DATASETS}

\section{A. First Dataset}

The first real dataset we use in this paper is a subset of a hyperspectral image acquired over the University of Houston campus, Texas, USA, in June 2012. The image comprises 144 spectral bands in the $380 \mathrm{~nm}$ to $1050 \mathrm{~nm}$ region, and comes with a Lidar dataset acquired a day before over the same area, with the same spatial resolution $(2.5 \mathrm{~m})$. We are interested here in a $152 \times 108 \times 144$ subset of this image, acquired over Robertson stadium on the Houston Campus and its surroundings. Fig. 12 shows a RGB representation of the observed scene, as well as a high spatial resolution RGB image of the scene ${ }^{2}$.

We are comparing the same algorithms as before. In the absence of ground truth, we will only assess the results visu-

\footnotetext{
${ }^{2}$ Note that it was acquired at a different time with a few notable changes with respect to the dataset we are interested in (mainly parked cars).
} 
ally, and give the running times and reconstruction errors of each algorithm. The regularization parameters for this dataset are gathered in the supplementary material. For both synthetic datasets, the PLMM algorithm was used with a spatial smoothness constraint on the abundances, and a constraint forcing the endmembers to be close the the reference extracted with VCA. The bundle used was extracted using 45 subsets of 2 percent of the pixels of the image, without replacement. For the proposed approach, we initialized the algorithm with the abundances of S-CLSU and the scaling factors set to one.

The estimated Intrinsic Dimensionality of the dataset using the Hysime algorithm [36] is 17, but we chose to consider only 4 endmembers. The reason for this is twofold: First, when 17 endmembers are extracted, for all algorithms, most abundance maps are very sparse and have very few spatial structure. This is either because outliers are selected as endembers or because a really rare material was chosen (such as an isolated car in a single pixel only). Besides, ID estimation algorithms provide an upper bound on the number of endmember to use, and the definition of an endmember is actually application and context dependent. Results with 4 endmembers are easier to visualize, to interpret and to compare for our endmember variability application. From the reference endmembers selected by VCA, we identified 4 classes: vegetation, concrete stands, asphalt and red metallic roofs. The vegetation endmember could have been splitted into grass and trees, but we chose to consider only one endmember vegetation to show the capability of the algorithms to recover an interpretable spectral variability. The same goes for the football field, which is actually mixed with soil. However, the soil endmember is very hard to extract, as there is probably no pure soil pixel for this material in the considered dataset.

1) Results: The results on the real dataset are shown in Fig. 13 for the abundances and in Fig. 14 for the spectral variability estimation. In addition, the reconstruction errors and running times of the different algorithms are shown in Table III. From Fig. 13, it seems that overall the abundance distributions of FCLSU follow the visual examination of the image, with very pure areas for the stands, and a good identification of the lawn in the stadium and of the stands (all the stands are indeed made of the same material if we refer to the high resolution RGB image, whereas it is not clear at all from the RGB composition of the hyperspectral data). However, the algorithm fails to consider the red metallic roofs as pure. The bundle approach combined with SUnSAL improves the purity of the red roof areas but the stands are not so well identified and interpreted as a mixture of concrete and asphalt. The CLSU algorithm (without scaling) obtains visually more coherent results, but both the red roofs and the concrete stands exhibit abundances which are significantly higher than 1 for all materials (up to 1.3, corresponding to saturated red values in Fig. 13), because CLSU does not actually estimate the abundances but a factor incorporating spectral variability. The FDN approach obtains very clear abundance maps for the vegetation and red roofs, but once again the distinction between concrete stands and asphalt (roads and parking lots) is not so clear. The abundances from the PLMM are visually not very satisfying since there are a lot a pixels which should be pure but are considered

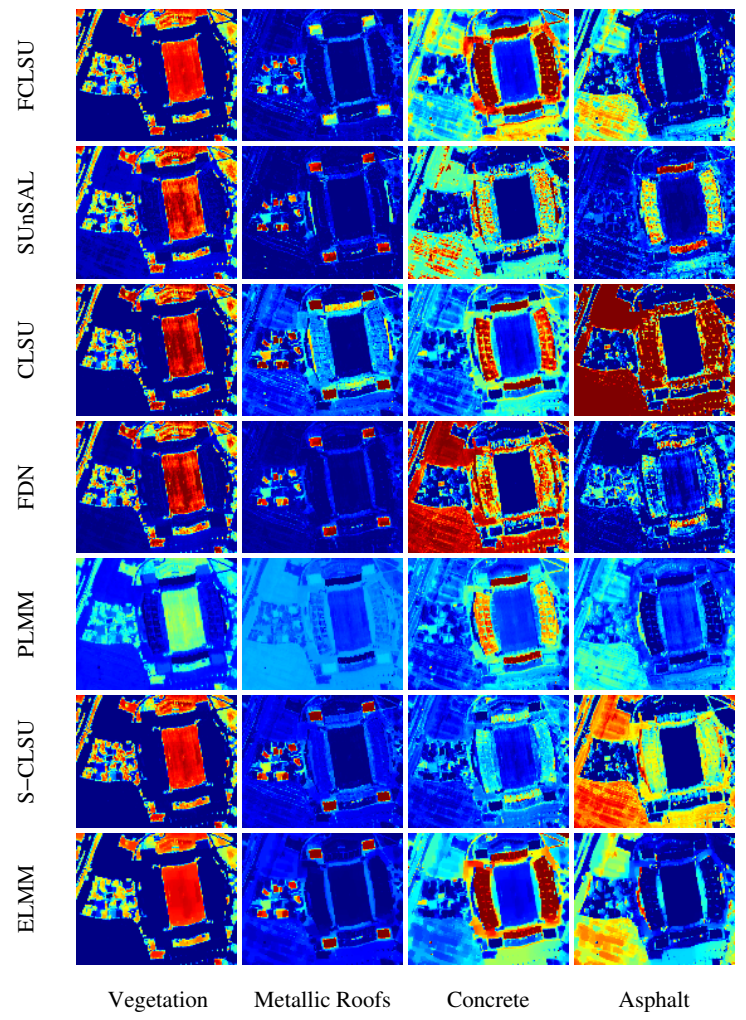

Fig. 13. The abundance maps estimated by all algorithms for the Houston dataset. The color scale goes from 0 (blue) to 1 (red). For CLSU, all the abundances higher than 1 are shown in red.

heavily mixed. However, the algorithm is still able to detect most of the areas where spectral variability occurs (red roofs, stadium stands for instance). S-CLSU, on the other hand, obtains visually good results since it corrects the estimations of CLSU thanks to the scaling. Then, the abundance maps for the red metallic roofs are better defined. The vegetation is also well identified. Asphalt and concrete stands are harder to discriminate. Besides, the scaling factors map is hard to interpret because only one scaling factor is estimated for all four endmembers. In Fig. 14, the color scale for each material was chosen using the results of the proposed approach. Otherwise, the results from S-CLSU have a very large dynamic, which makes it hard to visualize the results, added to the fact that there is only a single variability map for all materials. The proposed approach, although more computationally intensive, obtains visually good results as well. The vegetation is well identified, and the football field appears purer than with $S$ CLSU thanks to the spatial regularization. With our definitions of the endmembers, potential mixtures of grass with soil are interpreted as variability. The distinction between grass and tree leaves is also clearly identifiable in the scaling factors maps because the leaves areas are associated in this case to scaling factors smaller than 1 , even though in some cases the pixels can be mixed with red roofs or asphalt (for instance in the area to the left of the stadium). In this case, the model seems to accommodate the intrinsic variability of the materials since it can estimate different scaling factors for different materials in mixed pixels, thanks to the inclusion 


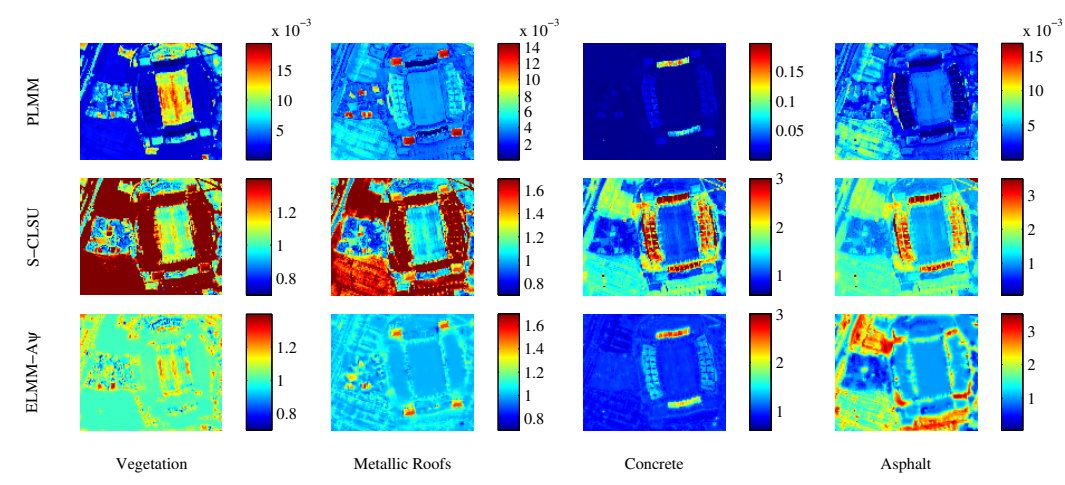

Fig. 14. Magnitude of the PLMM variability term (top row), the scaling factors estimated by S-CLSU (middle row) and the proposed approach (bottom row) for the Houston dataset.

\begin{tabular}{|c|c|c|c|c|c|c|c|}
\hline Algorithm & FCLSU & BUNDLES + SUnSAL & BUNDLES + FDN & CLSU & PLMM & S-CLSU & ELMM-A $\psi$ \\
\hline xRMSE & 0.0212 & 0.0065 & 0.0645 & 0.0047 & 0.0263 & 0.0047 & 0.0031 \\
\hline XSAM (degrees) & 3.3057 & 1.0953 & 4.1885 & 1.4531 & 6.6727 & 1.4531 & 0.9885 \\
\hline Running Time (s) & 4 & 6 & 5 & 4 & 333 & 5 & 402 \\
\hline
\end{tabular}

TABLE III

RUNNING TIMES AND RECONSTRUCTION ERRORS OF THE TESTED ALGORITHMS ON THE HOUSTON DATASET.

of one scaling factor for each material and to the spatial regularizations. The red roofs are also well detected, and the corresponding scaling factors significantly differ depending on the orientation of the roof (which we can clearly see with the high resolution image and the Lidar data). The same phenomenon occurs with the stands: the 4 stands are related to the same endmember class, but they all have significantly different scaling factors, depending once again on their orientation (they also correspond to significant elevation changes, as can be seen on the Lidar image). These two facts suggest that the ELMM is indeed able to identify variability due to changing illumination conditions. The asphalt abundance map coincides with the location of the parking lots, and also shows local variations in scale. Finally, the spatial regularization also eliminates outliers (cars) in the spatial distribution of the scaling factors and abundance maps.

\section{B. Second Dataset}

The second dataset we consider is a $200 \times 200 \times 186$ subset of the Cuprite dataset, which is shown in Fig. 15. The image was acquired by NASA's AVIRIS sensor and covers the Cuprite mining district in western Nevada, USA. We extracted 14 endmembers with the VCA according to the ID value estimated by Hysime on our subset. We compare the same algorithms as before and show in Fig. 16 the estimated abundance maps. The results are shown only for some of the extracted endmembers. For the concerned algorithms, we also show in Fig. 17 a map of the estimated spectral variability. We also show the reconstruction errors and the running times of all algorithms in Table IV. The materials have been identified by visual comparison between the estimated abundance maps and endmember signatures to those recovered in [15].

1) Results: From the visual results, we see for instance that FCLSU detects a near pure area of Alunite in the top of the rightmost part of the image, while this is interpreted as near pure Muscovite with variability by the ELMM and S-CLSU, which shows taking variability into account can significantly

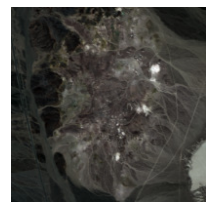

Fig. 15. A RGB representation of the subset of the Cuprite dataset used.

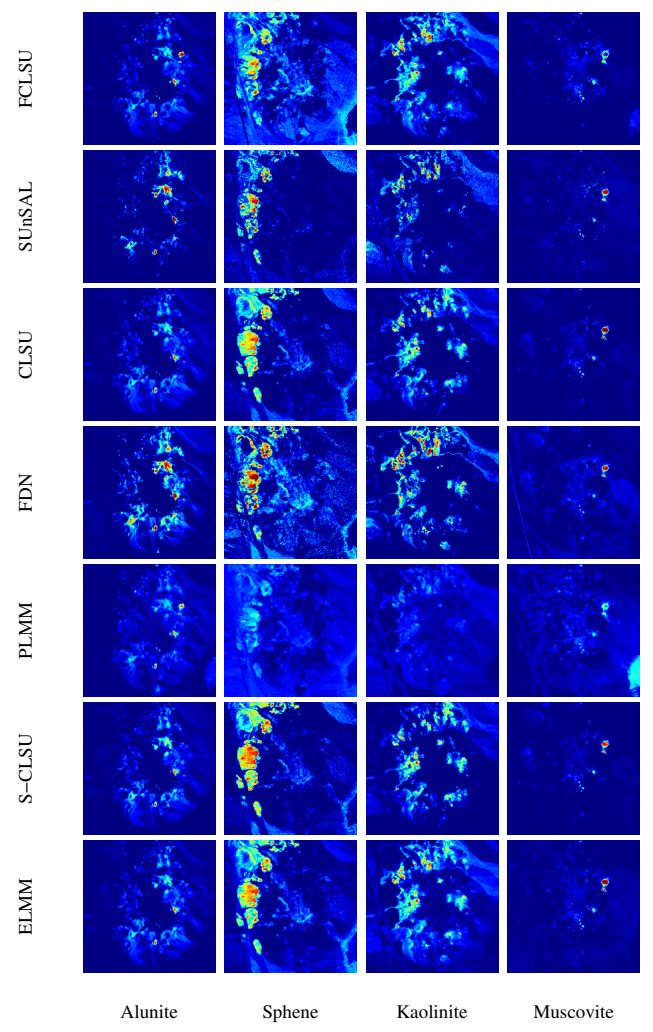

Fig. 16. The abundance maps estimated by some algorithms for the Cuprite dataset. The color scale goes from 0 (blue) to 1 (red). 


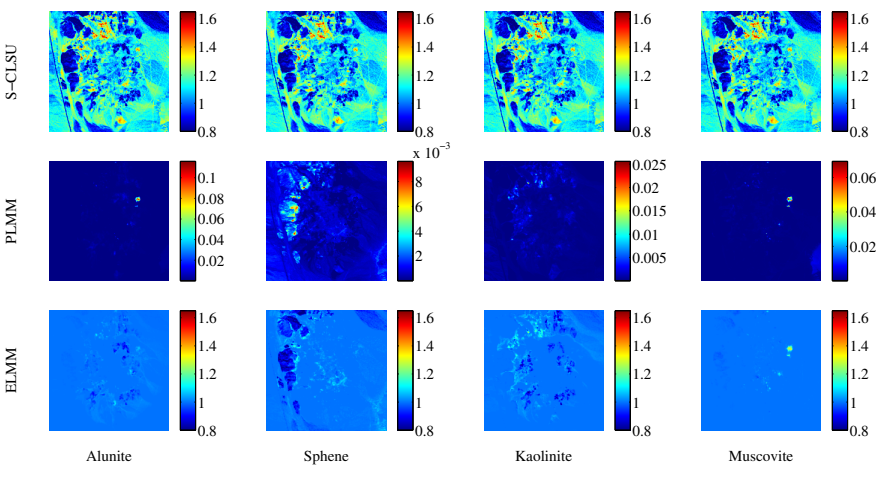

Fig. 17. Magnitude of the PLMM variability term (top row), the scaling factors estimated by S-CLSU (middle row) and the proposed approach (bottom row) for the Cuprite dataset.

change the abundance results. The PLMM algorithm detects more or less the same variability areas than the ELMM, but its abundance maps are in average lower, meaning that is interprets the data as being more mixed. As for the variability maps, for ELMM and S-CLSU, we chose the reference color scale to reflect the dynamic of the map of S-CLSU. This shows that for 14 materials, it can become very hard to interpret visually, and even more so for mixed pixels, while the ELMM results with one scaling factor for each material is much clearer, even at this scale, which favors S-CLSU. Anyway, we see that the materials in the scene seem to be significantly affected by spectral variability, which makes the abundance maps recovered by the algorithms taking it into account very different from the ones recovered by the usual LMM.

\section{CONCLUSION}

In this paper, we introduced an algorithm to unmix hyperspectral data according to a new Extended Linear Mixing Model. This geometrically interpretable model aims at incorporating spectral variability in the spectral unmixing problem under the form of scaled variations of reference endmembers, while preserving the framework of the usual Linear Mixing Model. We showed that the common partially Constrained Least Squares Unmixing algorithm can be interpreted in this framework, by assuming identical scalings for all endmembers. We also proposed a more sophisticated algorithm to unmix hyperspectral data following this model. The experiments on two synthetic datasets show how the proposed algorithm is able to take into account the spatial information provided by neighboring pixels thanks to a spatial regularization on the fractional abundances and on its spectral variability parameters. In addition, the algorithm is robust to the absence of pure pixels in the scene as well as to noise and deviations from the ELMM. Tests on two simulated hyperspectral dataset using abundance maps mimicking natural landscapes, and for one of them a realistic physics-based model to simulate the effect of changing illumination conditions because of the topography of the scene show that the ELMM is suited to address this kind of spectral variability. We compared the proposed algorithm to the scaled version of CLSU, and to other approaches of the literature which take spectral variability into account, and evidenced its superior performance in our simulations, both in terms of abundance estimation and spectral variability retrieval. Tests on real datasets show the potential of the model and algorithm to explain variability in practical scenarios. Future work will focus on finding an automated way to select the regularization parameters. We also plan to make more use of Lidar data by being able to recover the acquisition angles from the position of the sun and sensor, in order to match them to the estimated scaling factors.

\section{ACKNOWLEDGMENTS}

The authors would like to thank Rubén Marrero and Prof. Sylvain Douté from IPAG (Institut de Planétologie et d'Astrophysique de Grenoble) for providing the Hapke model code and the endmember data used in the second simulation, and also the Hyperspectral Image Analysis group and the NSF Funded Center for Airborne Laser Mapping at the University of Houston for providing the Houston dataset used in this study. Finally, the authors thank Pierre-Antoine Thouvenin from IRIT (Institut de Recherche en Informatique de Toulouse) for running the PLMM algorithm on the datasets used in this paper.

\section{REFERENCES}

[1] J. Bioucas-Dias, A. Plaza, G. Camps-Valls, P. Scheunders, N. Nasrabadi, and J. Chanussot, "Hyperspectral remote sensing data analysis and future challenges," Geoscience and Remote Sensing Magazine, IEEE, vol. 1, pp. 6-36, June 2013.

[2] M. Veganzones, G. Tochon, M. Dalla-Mura, A. Plaza, and J. Chanussot, "Hyperspectral image segmentation using a new spectral unmixingbased binary partition tree representation," Image Processing, IEEE Transactions on, vol. 23, pp. 3574-3589, Aug 2014.

[3] G. Camps-Valls, D. Tuia, L. Bruzzone, and J. Atli Benediktsson, "Advances in hyperspectral image classification: Earth monitoring with statistical learning methods," Signal Processing Magazine, IEEE, vol. 31, pp. 45-54, Jan 2014.

[4] N. Nasrabadi, "Hyperspectral target detection : An overview of current and future challenges," Signal Processing Magazine, IEEE, vol. 31, pp. 34-44, Jan 2014.

[5] T. Akgun, Y. Altunbasak, and R. Mersereau, "Super-resolution reconstruction of hyperspectral images," IEEE Trans. on Image Proc, pp. 1860-1875, 2005.

[6] W.-K. Ma, J. Bioucas-Dias, T.-H. Chan, N. Gillis, P. Gader, A. Plaza, A. Ambikapathi, and C.-Y. Chi, "A signal processing perspective on hyperspectral unmixing: Insights from remote sensing," Signal Processing Magazine, IEEE, vol. 31, pp. 67-81, Jan 2014.

[7] J. Bioucas-Dias, A. Plaza, N. Dobigeon, M. Parente, Q. Du, P. Gader, and J. Chanussot, "Hyperspectral unmixing overview: Geometrical, statistical, and sparse regression-based approaches," Selected Topics in Applied Earth Observations and Remote Sensing, IEEE Journal of, vol. 5, pp. 354-379, April 2012.

[8] R. Heylen, M. Parente, and P. Gader, "A review of nonlinear hyperspectral unmixing methods," Selected Topics in Applied Earth Observations and Remote Sensing, IEEE Journal of, vol. 7, pp. 1844-1868, June 2014.

[9] N. Dobigeon, J.-Y. Tourneret, C. Richard, J. Bermudez, S. McLaughlin, and A. Hero, "Nonlinear unmixing of hyperspectral images: Models and algorithms," Signal Processing Magazine, IEEE, vol. 31, pp. 82-94, Jan 2014.

[10] B. Somers, G. P. Asner, L. Tits, and P. Coppin, "Endmember variability in spectral mixture analysis: A review," Remote Sensing of Environment, vol. 115 , no. 7 , pp. 1603 - 1616, 2011.

[11] A. Zare and K. Ho, "Endmember variability in hyperspectral analysis: Addressing spectral variability during spectral unmixing," Signal Processing Magazine, IEEE, vol. 31, pp. 95-104, Jan 2014.

[12] O. Eches, N. Dobigeon, C. Mailhes, and J.-Y. Tourneret, "Bayesian estimation of linear mixtures using the normal compositional model. application to hyperspectral imagery," Image Processing, IEEE Transactions on, vol. 19, pp. 1403-1413, June 2010.

[13] X. Du, A. Zare, P. Gader, and D. Dranishnikov, "Spatial and spectral unmixing using the beta compositional model," Selected Topics in Applied Earth Observations and Remote Sensing, IEEE Journal of, vol. 7, pp. 1994-2003, June 2014. 


\begin{tabular}{|c|c|c|c|c|c|c|c|}
\hline Algorithm & FCLSU & BUNDLES + SUnSAL & BUNDLES + FDN & CLSU & PLMM & S-CLSU & ELMM-A $\psi$ \\
\hline xRMSE & 0.0062 & 0.0138 & 0.0361 & 0.0047 & 0.0086 & 0.0047 & 0.0034 \\
\hline xSAM (degrees) & 0.9026 & 0.4759 & 1.1954 & 0.7088 & 1.2708 & 0.7088 & 0.5112 \\
\hline Running Time (s) & 26 & 96 & 30 & 27 & $4.10^{3}$ & 28 & 647 \\
\hline
\end{tabular}

TABLE IV

RUNNING TIMES AND RECONSTRUCTION ERRORS OF THE TESTED ALGORITHMS ON THE CUPRITE DATASET.

[14] J. Plaza, E. Hendrix, I. García, G. Martín, and A. Plaza, “On endmember identification in hyperspectral images without pure pixels: A comparison of algorithms," Journal of Mathematical Imaging and Vision, vol. 42, no. 2-3, pp. 163-175, 2012.

[15] J. Nascimento and J. Bioucas Dias, "Vertex component analysis: a fast algorithm to unmix hyperspectral data," Geoscience and Remote Sensing, IEEE Transactions on, vol. 43, pp. 898-910, April 2005.

[16] M. E. Winter, "N-findr: an algorithm for fast autonomous spectral endmember determination in hyperspectral data," in Proc. SPIE, vol. 3753, pp. 266-275, 1999.

[17] J. Li and J. Bioucas-Dias, "Minimum volume simplex analysis: A fast algorithm to unmix hyperspectral data," in Geoscience and Remote Sensing Symposium, 2008. IGARSS 2008. IEEE International, vol. 3, pp. III - 250-III - 253, July 2008.

[18] R. Ammanouil, A. Ferrari, C. Richard, and D. Mary, "Blind and fully constrained unmixing of hyperspectral images," Image Processing, IEEE Transactions on, vol. 23, pp. 5510-5518, Dec 2014.

[19] Y. Altmann, N. Dobigeon, and J.-Y. Tourneret, "Unsupervised postnonlinear unmixing of hyperspectral images using a hamiltonian monte carlo algorithm," Image Processing, IEEE Transactions on, vol. 23, pp. 2663-2675, June 2014.

[20] M. Veganzones, L. Drumetz, R. Marrero, G. Tochon, M. Dalla Mura, A. Plaza, J. Bioucas-Dias, and J. Chanussot, "A new extended linear mixing model to address spectral variability," in Proc. IEEE Workshop on Hyperspectral Image and Signal Processing: Evolution in Remote Sensing (WHISPERS), 2014.

[21] P.-A. Thouvenin, N. Dobigeon, and J.-Y. Tourneret, "Hyperspectral unmixing with spectral variability using a perturbed linear mixing model," Signal Processing, IEEE Transactions on, vol. 64, no. 2, pp. 525-538, 2016.

[22] B. Somers, M. Zortea, A. Plaza, and G. Asner, "Automated extraction of image-based endmember bundles for improved spectral unmixing," Selected Topics in Applied Earth Observations and Remote Sensing, IEEE Journal of, vol. 5, pp. 396-408, April 2012.

[23] M.-D. Iordache, J. Bioucas-Dias, and A. Plaza, "Sparse unmixing of hyperspectral data," Geoscience and Remote Sensing, IEEE Transactions on, vol. 49, pp. 2014-2039, June 2011.

[24] J. Jin, B. Wang, and L. Zhang, "A novel approach based on fisher discriminant null space for decomposition of mixed pixels in hyperspectral imagery," Geoscience and Remote Sensing Letters, IEEE, vol. 7, pp. 699-703, Oct 2010.

[25] D. Heinz and C.-I. Chang, "Fully constrained least squares linear spectral mixture analysis method for material quantification in hyperspectral imagery," Geoscience and Remote Sensing, IEEE Transactions on, vol. 39, pp. 529-545, Mar 2001.

[26] J. Nascimento and J. Bioucas Dias, "Does independent component analysis play a role in unmixing hyperspectral data?," Geoscience and Remote Sensing, IEEE Transactions on, vol. 43, pp. 175-187, Jan 2005.

[27] X. C. Garcia, Evaluation des performances de l'analyse statistique et physique d'images hyperspectrales de Mars. Application au capteur multi-angulaire CRISM (In English). PhD thesis, Université de Grenoble, December 2011.

[28] N. Keshava and J. F. Mustard, "Spectral unmixing," Signal Processing Magazine, IEEE, vol. 19, no. 1, pp. 44-57, 2002.

[29] B. Hapke, Theory of reflectance and emittance spectroscopy. Cambridge University Press, 2012.

[30] M. Kowalski, K. Siedenburg, and M. Dorfler, "Social sparsity! neighborhood systems enrich structured shrinkage operators," Signal Processing, IEEE Transactions on, vol. 61, no. 10, pp. 2498-2511, 2013.

[31] M.-D. Iordache, J. Bioucas-Dias, and A. Plaza, "Total variation spatial regularization for sparse hyperspectral unmixing," Geoscience and Remote Sensing, IEEE Transactions on, vol. 50, pp. 4484-4502, Nov 2012.

[32] C. Bilen, G. Puy, R. Gribonval, and L. Daudet, "Convex optimization approaches for blind sensor calibration using sparsity," Signal Processing, IEEE Transactions on, vol. 62, pp. 4847-4856, Sept 2014.

[33] S. Boyd, N. Parikh, E. Chu, B. Peleato, and J. Eckstein, "Distributed optimization and statistical learning via the alternating direction method of multipliers," Foundations and Trends in Machine Learning, vol. 3, no. 1, pp. 1-122, 2011.

[34] A. M. Cord, P. C. Pinet, Y. Daydou, and S. D. Chevrel, "Experimental determination of the surface photometric contribution in the spectral reflectance deconvolution processes for a simulated martian crater-like regolithic target," Icarus, vol. 175, no. 1, pp. 78 - 91, 2005.

[35] R. Marrero, S. Douté, A. Plaza, and J. Chanussot, "Validation of spectral unmixing methods using photometry and topography information," in Proc. IEEE Workshop on Hyperspectral Image and Signal Processing: Evolution in Remote Sensing (WHISPERS), 2013.

[36] J. Bioucas-Dias and J. Nascimento, "Hyperspectral subspace identification," Geoscience and Remote Sensing, IEEE Transactions on, vol. 46, pp. 2435-2445, Aug 2008.

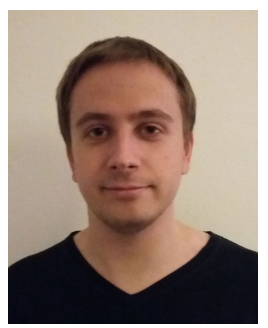

Lucas Drumetz received the M.Sc degree in Electical Engineering from the Grenoble Institute of Technology (Grenoble-INP), France, in 2013. Since then, he has been working towards the $\mathrm{PhD}$ degree in signal and image processing at the University of Grenoble-Alpes. His research interests focus on spectral unmixing of hyperspectral data, and on the applications of optimization techniques to hyperspectral images and remote sensing. He is a reviewer for the IEEE JOURNAL OF SELECTED TOPICS IN APPLIED EARTH OBSERVATIONS AND Remote Sensing and the IEEE Geoscience And Remote SEnsing LETTERS.

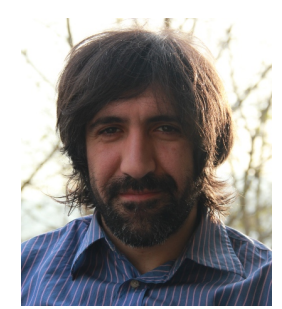

Miguel A. Veganzones (M'12) received the M.Sc. and Ph.D. degrees in computer science from the Basque Country University (UPV/EHU), Donostia, Spain, in 2005 and 2012 respectively. Since 2012, he is a posdoctoral researcher at the Images-Signal department in the GIPSA-lab, Grenoble, France. His current main research activities focus in the analysis of hyperspectral images by means of computational intelligence, statistical techniques and tensor data analysis. He is a Reviewer of IEEE TRANSACTIONS ON IMAGE PROCESSING, IEEE TRANSACTIONS ON GEOSCIENCE AND REMOTE SENSING, IEEE GEOSCIENCE AND Remote Sensing LetTers, IEEE Journal of Selected Topics in APPLIED EARTH OBSERVATIONS AND REMOTE SENSING and the IEEE Journal of SElEcted Topics in Signal Processing.

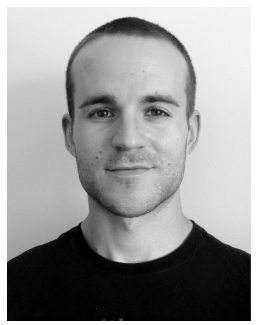

Simon Henrot was born in France in 1986. He graduated in electrical engineering from the Ecole Nationale de l'Aviation Civile, Toulouse, France in 2009 and received the Ph.D. degree in signal processing from the Université de Lorraine, Nancy, France in 2013. He is currently a post-doctoral student with Université Joseph Fourier, Grenoble, France at the Grenoble Images Speech Signals and Automatics Laboratory (GIPSA-Lab). His research interest include inverse problems in imaging and multimodal image processing. 


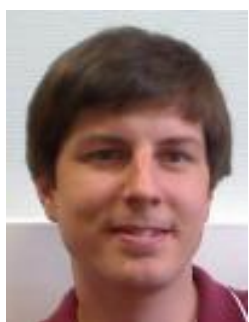

Ronald Phlypo was born in Belgium in 1981. He received the M.Eng. degree in electronical engineering from KHBO, Ostend, Belgium in 2003 and a M.Sc. degree in Artificial Intelligence from KULeuven, Leuven, Belgium in 2004. He obtained his doctoral degree in engineering from the University of Ghent, Belgium in 2009. From 2009 to 2012, he was with GIPSA lab in Grenoble, France, as a post-doctoral research associate working on a signal processing model for joint Gaze-EEG recordings. From 2012 to 2013, he has been an associate researcher at the machine learning for signal processing lab in Baltimore, Maryland, US, where he has worked on linear latent variable models for multimodality. In 2013, he has held an engineering position at the INRIA institute in Saclay, France, where he was involved in the untanglement of functional magnetic resonance imaging cognitive connectivity estimates. Since 2014, he holds an associate professor position at PHELMA, Grenoble Institute of technology (Grenoble INP), Grenoble, France, with a research component at GIPSA-lab, Grenoble, France. His current research interests are on functional connectivity estimates, multimodality, and multistability with applications to biomedical signal processing.

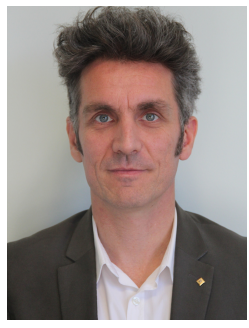

Jocelyn Chanussot (M'04-SM'04-F'12) received the M.Sc. degree in electrical engineering from the Grenoble Institute of Technology (Grenoble INP), Grenoble, France, in 1995, and the Ph.D. degree from Savoie University, Annecy, France, in 1998. In 1999, he was with the Geography Imagery Perception Laboratory for the Delegation Generale de l'Armement (DGA - French National Defense Department). Since 1999, he has been with Grenoble INP, where he was an Assistant Professor from 1999 to 2005, an Associate Professor from 2005 to 2007, and is currently a Professor of signal and image processing. He is conducting his research at the Grenoble Images Speech Signals and Automatics Laboratory (GIPSA-Lab). His research interests include image analysis, multicomponent image processing, nonlinear filtering, and data fusion in remote sensing. He has been a visiting scholar at Stanford University (USA), KTH (Sweden) and NUS (Singapore). Since 2013, he is an Adjunct Professor of the University of Iceland. In 2014-2015, he is a visiting professor at the University of California, Los Angeles (UCLA). Dr. Chanussot is the founding President of IEEE Geoscience and Remote Sensing French chapter (20072010) which received the 2010 IEEE GRS-S Chapter Excellence Award. He was the co-recipient of the NORSIG 2006 Best Student Paper Award, the IEEE GRSS 2011 and 2015 Symposium Best Paper Award, the IEEE GRSS 2012 Transactions Prize Paper Award and the IEEE GRSS 2013 Highest Impact Paper Award. He was a member of the IEEE Geoscience and Remote Sensing Society AdCom (2009-2010), in charge of membership development. He was the General Chair of the first IEEE GRSS Workshop on Hyperspectral Image and Signal Processing, Evolution in Remote sensing (WHISPERS). He was the Chair (2009-2011) and Cochair of the GRS Data Fusion Technical Committee (2005-2008). He was a member of the Machine Learning for Signal Processing Technical Committee of the IEEE Signal Processing Society (2006-2008) and the Program Chair of the IEEE International Workshop on Machine Learning for Signal Processing, (2009). He was an Associate Editor for the IEEE Geoscience and Remote Sensing Letters (2005-2007) and for Pattern Recognition (2006-2008). Since 2007, he is an Associate Editor for the IEEE Transactions on Geoscience and Remote Sensing. He was the Editor-inChief of the IEEE Journal of Selected Topics in Applied Earth Observations and Remote Sensing (2011-2015). In 2013, he was a Guest Editor for the Proceedings of the IEEE and in 2014 a Guest Editor for the IEEE Signal Processing Magazine. He is a Fellow of the IEEE and a member of the Institut Universitaire de France (2012-2017).

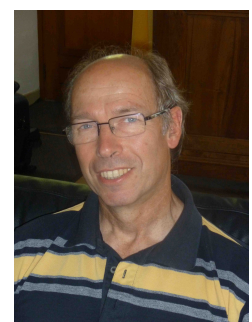

Christian Jutten (AM'92-M'03-SM'06-F'08) received h.D. and Doctor ès Sciences degrees in signal processing from Grenoble Institute of Technology (GIT), France, in 1981 and 1987, respectively. From 1982, he was an Associate Professor at GIT, before being Full Professor at University Joseph Fourier of Grenoble, in 1989. For 35 years, his research interests have been machine learning and source separation, including theory (separability, source separation in nonlinear mixtures, sparsity, multimodality) and applications (brain and hyperspectral imaging, chemical sensor array, speech). He is author or coauthor of more than 90 papers in international journals, 4 books, 25 keynote plenary talks and about 200 communications in international conferences. He has been visiting professor at Swiss Federal Polytechnic Institute (Lausanne, Switzerland, 1989), at Riken labs (Japan, 1996) and at Campinas University (Brazil, 2010). He was director or deputy director of his lab from 1993 to 2010, especially head of the signal processing department (120 people) and deputy director of GIPSA-lab (300 people) from 2007 to 2010 . He was a scientific advisor for signal and images processing at the French Ministry of Research (1996-1998) and for the French National Research Center (2003-2006). From May 2012 to September 2014, he was deputy director at the Institute for Information Sciences (INS2I) at French National Center of Research (CNRS) in charge of signal and image processing. Christian Jutten was organizer or program chair of many international conferences, especially of the 1st International Conference on Blind Signal Separation and Independent Component Analysis in 1999. He has been a member of a few IEEE Technical Committees, and currently in "SP Theory and Methods" of the IEEE Signal Processing society. He received best paper awards of EURASIP (1992) and of IEEE GRSS (2012), and Medal Blondel (1997) from the French Electrical Engineering society for his contributions in source separation and independent component analysis. $\mathrm{He}$ is IEEE fellow (2008) and EURASIP fellow (2013). He is a Senior Member of the Institut Universitaire de France since 2008, with renewal in 2013 for 5 years. He is the recipient of a 2012 ERC Advanced Grant for a project on challenges in extraction and separation of sources (CHESS). 